\title{
Production Linkage of Asia and Europe via Central and Eastern Europe
}

\author{
Mitsuyo Ando \\ Keio University, Tokyo, Japan \\ Fukunari Kimura \\ Keio University, Tokyo, Japan
}

\begin{abstract}
This paper investigates the novel development of machinery trade pattern in the Central and Eastern Europe (CEE) and analyzes the extent and depth of production networks in Europe from the perspective of their links with East Asia during the past 15 years, 1995 2010. Our descriptive analysis based on the total value of trade and the extensive margin demonstrates further production fragmentation in Europe, shifting from Western Europe (CEE production sharing to a regional network with more active transactions among CEE countries) to a global link with East Asia via CEE, particularly in the electric machinery sector. Our quantitative analysis verifies the evolution of production networks in Europe, from regional to global. These results partially reflect the reduction in services link costs, the evolution of industrial clustering among CEE countries particularly after the EU enlargement, and the strengthening competence of production networks in East Asia.
\end{abstract}

JEL Classifications: F14, F15, F23, L23

Key Words: The 2nd unbundling, Fragmentation, Agglomeration, Intensive and Extensive Margins, Machinery trade

\footnotetext{
* Corresponding Author: Mitsuyo Ando; Faculty of Business and Commerce, Keio University, 2-15-45 Mita, Minato-ku, Tokyo 1088345, Japan; Tel: +81 334534511, Fax: +81 354271578, E-mail: m-ando@fbc.keio.ac.jp.

Co-Author: Fukunari Kimura; Faculty of Economics, Keio University, 2-15-45 Mita, Minato-ku, Tokyo 108-8345, Japan; Tel: +81 334534511, Fax: +81 354271578, E-mail: fkimura@econ.keio.ac.jp.

Acknowledgements: The authors would like to acknowledge anonymous referees for valuable comments.
} 


\section{Introduction}

The 2nd unbundling, that is, international division of labor in terms of geographically separating various production stages, has gradually dominated the world trade since the 1980s (Baldwin, 2011). Now the second unbundling is coming into a new phase in terms of the geographical extension of production networks from regional to global.

The 2nd unbundling in the manufacturing sector, particularly in machinery industries, has been developed in three regions: the US-Mexico/US-Costa Rica cross-border production sharing, the Western Europe (WE) and the Central and Eastern Europe (CEE) manufacturing nexus, and the East Asian production networks. The degree of complexity and sophistication of the 2nd unbundling differs across the regions. Cross-border production sharing in the Americas mostly remains simplistic intra-firm, back-and-forth division of labor across national borders without meaningful linkages with developing countries' domestic economies. ${ }^{1}$ In East Asia, regional production networks have reached further sophistication where many countries/regions get involved, fine-tuning combination of intra-firm and inter-firm (arm's length) transactions is developed, and the formation of industrial agglomerations on the developing countries' side is advanced. ${ }^{2}$ By the beginning of the 2000s, East Asia established dominance in electronics parts and components.

CEE, of which Czech Republic (Czech hereinafter) and Hungary played key roles, started from a relatively simplistic cross-border production sharing with WE. Their European Union (EU) accession, however, provided a chance of a substantial break-through. ${ }^{3}$ The EU enlargement changed the rules of origin and tariff structure and provided CEE a possibility of more flexible operations including transactions among CEE countries with lower tariffs due to their EU accession. In addition, firms began to conduct the final stage of production in CEE; before 2004, tariffs imposed on some final products were high in the EU, and thus the final stage of production used to be done in WE. After the EU enlargement in 2004, keeping tight relationships with WE, CEE started deepening the involvement in production networks and forming industrial agglomeration across CEE with stronger links with East Asia, particularly in machinery parts and components. That was the moment when geographical extension of production networks was enlarged from regional and global. Some scholars use the word "global supply/ value chains" where both production processes and wholesale/retail sales are included. However, trade in final products and trade in intermediate goods are qualitatively different. Final prod-

\footnotetext{
${ }^{1}$ Ando, Arndt, and Kimura (2009) demonstrated these evidences, using the firm level data of the Japanese and U.S. firms in Latin America, mainly in Mexico.

2 See, for instance, Ando and Kimura $(2005,2009)$ for sophisticated features of production networks in East Asia and Ando (2006) for the rapid development of trade patterns with vertical transactions of machinery parts and components in the region in the 1990s.

${ }^{3}$ Czech, Estonia, Cyprus, Latvia, Lithuania, Hungary, Malta, Poland, Slovakia, and Slovenia joined the EU in May 2004, and Romania and Bulgaria joined in January 2007. See the website for the details: http://ec.europa.eu/enlargement/policy/from-6-to-27members/index_en.htm.
} 
ucts are not usually perishable in the short run, and thus time costs and reliability of logistics links are not essential to their trade. On the other hand, trade in intermediate goods, particularly trade in parts and components, is very sensitive to not only monetary costs but also time costs and reliability of logistic links. In this sense, the recent phenomenon in the CEE region is worth being investigated in serious research.

The purpose of this paper is to employ international trade data in the disaggregated commodity classification and to trace the development of international production networks in Europe from the perspective of a link between and CEE and East Asia. The paper also discusses a number of questions related to CEE's industrial development. How quickly has each CEE country participated in production networks? How has East Asia come into production networks that extends from the east end to the west end in the largest continent in the world? How has the dominance of East Asia on electronic parts and components affected the formation of such production networks? How far has the formation of industrial agglomeration advanced in CEE, particularly in automobile industry?

\section{Fragmentation and Agglomeration}

Production networks, particularly in manufacturing processes, are regional in nature. The fragmentation theory suggests that services link to connect remotely located production blocks is the key in the formation and operation of production networks. ${ }^{4}$ Thus, production blocks are not supposed to be placed too far in general.

In the 1st unbundling, trade in raw materials and finished products is not very sensitive to time costs and thus can occur at a long distance. Trade in parts and components in the 2nd unbundling is considerably different in nature. ${ }^{5}$ Parts and components must be procured to each production block in the timely manner. A slight delay in the arrival of one single part may stop the whole production lines. Subtle coordination is required among multiple production blocks and service links that connect each production block are crucial, with tight Information and Communication Technology (ICT) and logistics connectivity. Therefore, a large portion of machinery parts and components trade occurs in general within a region such as East Asia.

The recent years, however, have witnessed a new phenomenon: significant links between East Asian and European production networks through CEE. Production networks across the largest continent emerge.

Figures 1 and 2 present the ratios of machinery goods in total exports to and imports from

\footnotetext{
${ }^{4}$ See, for instance, Jones and Kierzkowski (1990), Arndt and Kierzkowski (2001), and Deardorff (2001) for the fragmentation theory.

${ }^{5}$ As for the concept of the 1st and 2nd unbundling, see Baldwin (2011).
} 
the world in selected countries in 1995 and 2010; they array countries in terms of the export share of machinery parts and components. ${ }^{6}$ The portion of machinery parts and components is shown with stripes. ${ }^{7}$ The ratios of machinery parts and components exports are in particular useful indicators to judge how far the concerned country participates in international production networks in machinery industries. In 1995, the Philippines, Singapore, Malaysia, Korea, Thailand and Hong Kong were already involved in tight production networks in East Asia, with high ratios of parts and components for both exports and imports suggesting active back-andforth transactions, though China takes a bit of more time to come in. Among CEE, Hungary and Czech were obvious participants in international production networks by 1995 . Hungary and Czech used to be a part of cross-border production sharing in Europe, similar to the one between the US and Mexico; basically, they make assemblies, using parts and components that are procured by WE, and send them back to WE. On the other hand, Slovakia, Poland, and Romania were still lagging behind in joining international production networks. The positioning of CEE, however, changed drastically by 2010; these five CEE countries become active players in international production networks with higher ratios of machinery parts and components exports at around or more than 20 percent.

\footnotetext{
${ }^{6}$ To examine whether one can see different patterns between before and after the Global Financial Crisis, the corresponding pattern in 2007 was also investigated. The patterns in 2007 and 2010 do not show a significant difference.

Machinery is defined as HS84-92. Machinery parts and components are as follows: $8406, \quad 8407, \quad 8408, \quad 8409, \quad 8410$,

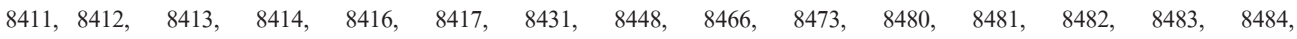

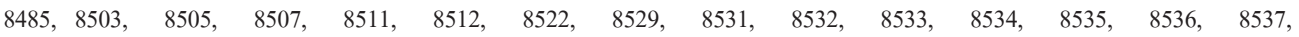

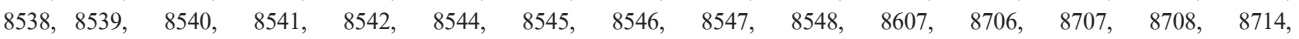
$8803,8805, \quad 9001, \quad 9002, \quad 9003, \quad 9013,9014,9033,9104,9110,9111,9112,9113, \quad 9114, \quad 9209$, $840140,840290,840390,840490,840590,841590,841891,841899,841990,842091,842099,842123,842129,842131$, $842191,842199,842290,842390,842490,843290,843390,843490,843590,843691,843699,843790,843890,843991$, $843999,844090,844190,844240,844250,844390,845090,845190,845240,845290,845390,845490,845590,846791$, $846792,846799,846890,847490,847590,847690,847790,847890,847990,850490,850690,850890,850990,851090$, $851390,851490,851590,851690,851790,851840,851850,851890,853090,854390,870990,871690,900590,900691$, 900699, 900791, 900792, 900890, 900990, 901090, 901190, 901290, 901590, 901790, 902490, 902590, 902690, 902790, 902890，902990，903090，903190，903290 (the version of HS2002 (See Ando and Kimura (2005)). Machinery final products are defined as those other than parts and components.
} 
Figure 1. Machinery goods and machinery parts and components: shares in total exports and imports in 1995

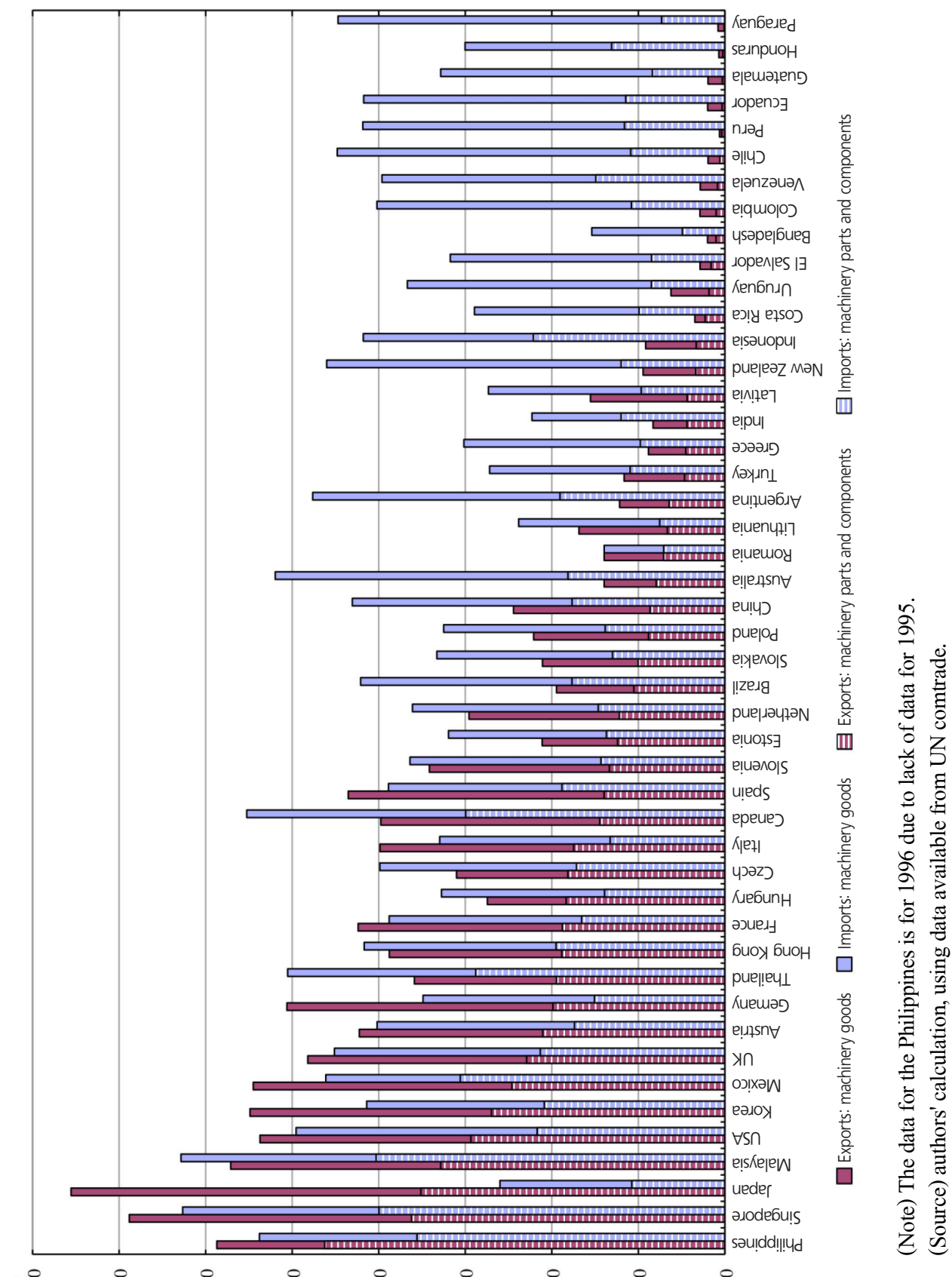


Figure 2. Machinery goods and machinery parts and components: shares in total exports and imports in 2010

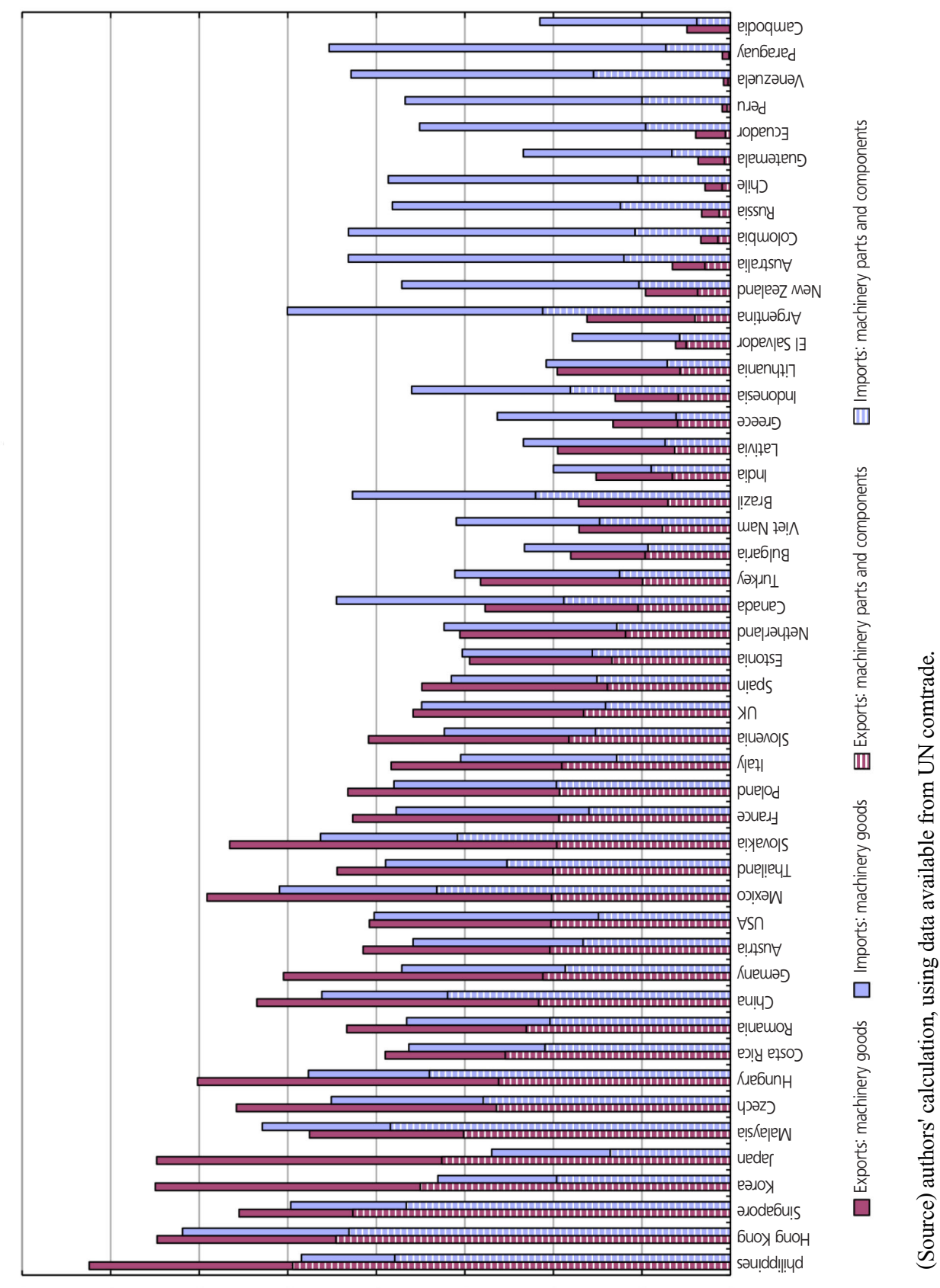


In the process of such development of production networks, particularly in the last decade East Asia seems to play an important role. Multinational enterprises (MNEs) in WE establish their manufacturing affiliates in CEE, and such moves are accelerated after the accession of CEE to the EU. In the dominance of East Asia in electronics industry, MNEs in WE start introducing massive electronic parts and components by importing from their affiliates and/or unrelated firms in East Asia to employ them for their production in CEE. Moreover, in forming industrial agglomerations in CEE, particularly in automobile industry, machinery parts and components imported from East Asia also play an important supplementary role. In addition, MNEs originated from East Asia such as Japan and Korea conduct foreign direct investment (FDI) in CEE and intensify sourcing from East Asia. ${ }^{8}$ These end up with tight production links between East Asia and CEE to serve for the market in WE, particularly after the EU enlargement. We observe the emergence of connectivity from the East end of the largest continents to the West end, bridged by CEE. It is worthwhile examining international trade data to trace the process of establishing a linkage between East Asia and Europe via CEE.

Another important feature in CEE is the geographical extension of industrial agglomeration. In East Asia, the formation of industrial agglomeration is motivated by the requirement of geographical proximity in inter-firm fragmentation (Kimura and Ando, 2005). Production networks typically start with intra-firm fragmentation in geographical distance. As production networks are increasingly sophisticated, however, inter-firm division of labor comes into the architecture. Intra-firm division of labor is typically in geographical proximity because transaction costs between unrelated firms have strong positive relationship with geographical distance. Typical industrial agglomerations such as Bangkok Metropolitan Area, Pearl River Basin, and Shanghai and its backyard have geographical extension of about $100 \mathrm{~km}$ diameter where 2 to 2.5 hour-drives by trucks cover the whole area with just-in-time production networks.

On the other hand, CEE seems to start with initial conditions that are different from East Asia. Population size of CEE countries is much smaller than typical East Asian countries. In addition, governments in CEE adopt long-standing policies to locate industrial activities in rural areas, rather than in urban vicinity. As a result, industrial agglomerations in CEE, for example in automobile industry, seem to be extended way beyond $100 \mathrm{~km}$ diameter, typically going beyond each country's national border. International trade data may capture such cross-border formation of industrial agglomerations in CEE.

\footnotetext{
${ }^{8}$ One of the most significant factors behind FDI in CEE by Japanese firms is the proximity to their customers in WE. Since parts and components are highly customized, and closer communication with suppliers is important particularly in Europe, firms need to locate nearby to conduct vertical and flexible adjustment. Japanese machinery affiliates in CEE often sell their parts and components to WE to be used in the final stage of production in WE considering the rules of origin of EU and high tariffs imposed on some final products. Particularly before the EU expansion, we observe this behavior of Japanese machinery affiliates in CEE instead of conducting the final stage in CEE(Ando and Kimura, 2007). The EU enlargement significantly changed locations of production blocks within Europe.
} 


\section{Evolution of Machinery Trade in CEE}

This section descriptively analyzes the developing patterns of machinery trade for CEE from 1995 to 2010 from the perspective of production networks in Europe and their links with East Asia via CEE.

\section{A. Features based on trade value}

Table 1 presents values of machinery trade for five major countries in CEE (CEE5 hereinafter) in 1995 and 2010 and the share of each CEE country among them, with a distinction of machinery parts and components and machinery final products, in (a) all machinery sectors (HS84 to 92), (b) electric machinery sector (HS85), and (c) transport equipment sector (HS86 to 89). CEE5 consist of five forerunners of CEE, i.e., Czech Republic, Hungary, Poland, and Slovakia, and Romania that has recently increased machinery trade shares. ${ }^{9}$ Table 2 (i) presents more detailed statistical figures on machinery import and export patterns of CEE5 in 1995 and 2010, that is, not only trade values to the world but also value index by major origins/destinations, i.e, the world, East Asia (Asia in the tables/figures), WE, and CEE, and shares of each origin/ destination in total trade (trade to the world) in (a) all machinery sectors, (b) electric machinery sector, and (c) transport equipment sector. The corresponding tables for each CEE5 are shown in Table 2 (ii) to 2 (vi). ${ }^{10}$

\footnotetext{
${ }^{9}$ As in Figure 1, Romania had a much lower portion of machinery goods in total exports and imports in 1995 among CEE5. In addition, while Romania has a larger Gross Domestic Product (GDP) than Slovakia with the smallest value of machinery trade among forerunners of CEE, the shares of Romania among CEE5 are the lowest on the export side; for instance, the share in 1995 are six percent (six percent) for machinery total (only parts and components), five percent (four percent) for electric machinery, and seven percent (five percent) for transport equipment. Although Romania obtained the larger shares in 2010, they are still less than 10 percent in most cases of exports. On the import side, the shares are more or less equal to those for Slovakia in 1995, but Romania still lagged behind Slovakia, particularly for imports of parts and components in 2010 .

${ }^{10}$ The paper defines East Asia as the nine East Asian countries (China, Hong Kong, Indonesia, Japan, Korea, Malaysia, Philippines, Singapore, and Thailand), WE as the six Western European countries (Austria, France, Germany, Italy, Netherland, and United Kingdom, and CEE as CEE5.
} 
Table 1. Machinery trade in CEE

\begin{tabular}{|c|c|c|c|c|c|c|c|c|c|c|c|c|}
\hline \multirow[b]{3}{*}{ Origin/destination } & \multicolumn{6}{|c|}{ Imports } & \multicolumn{6}{|c|}{ Exports } \\
\hline & \multicolumn{2}{|l|}{ Total } & \multicolumn{2}{|l|}{ Parts } & \multicolumn{2}{|l|}{ Final } & \multicolumn{2}{|l|}{ Total } & \multicolumn{2}{|l|}{ Parts } & \multicolumn{2}{|l|}{ Final } \\
\hline & 1995 & 2010 & 1995 & 2010 & 1995 & 2010 & 1995 & 2010 & 1995 & 2010 & 1995 & 2010 \\
\hline \multicolumn{13}{|c|}{ (a) All machinery sectors } \\
\hline \multicolumn{13}{|c|}{ Total value (millions US\$) } \\
\hline CEE5 & 30,005 & 216,980 & 12,730 & 131,300 & 17,275 & 85,680 & 18,064 & 256,220 & 9,659 & 113,500 & 8,405 & 142,720 \\
\hline \multicolumn{13}{|c|}{ Share in CEE 5 (total value $=100)$} \\
\hline Czech & 33.6 & 26.1 & 34.4 & 26.7 & 33.1 & 25.3 & 37.2 & 28.8 & 40.9 & 30.5 & 33.0 & 27.4 \\
\hline Hungary & 16.6 & 19.2 & 16.7 & 22.6 & 16.4 & 13.9 & 18.9 & 22.2 & 23.7 & 21.9 & 13.5 & 22.6 \\
\hline Poland & 31.4 & 30.5 & 31.7 & 26.0 & 31.3 & 37.4 & 28.0 & 26.5 & 20.9 & 26.6 & 36.1 & 26.3 \\
\hline Romania & 9.3 & 10.5 & 8.8 & 9.6 & 9.7 & 11.7 & 6.1 & 8.4 & 5.8 & 10.0 & 6.5 & 7.1 \\
\hline Slovakia & 9.1 & 13.7 & 8.4 & 15.1 & 9.6 & 11.7 & 9.8 & 14.1 & 8.7 & 11.0 & 11.0 & 16.6 \\
\hline \multicolumn{13}{|c|}{ (b) Electric machinery sector } \\
\hline \multicolumn{13}{|c|}{$\overline{\text { Total value (millions US\$) }}$} \\
\hline CEE5 & 8,076 & 91,080 & 5,218 & 63,150 & 2,858 & 27,930 & 5,608 & 97,955 & 4,293 & 41,730 & 1,315 & 56,225 \\
\hline \multicolumn{13}{|c|}{ Share in CEE5 (total value $=100)$} \\
\hline Czech & 35.4 & 24.1 & 36.4 & 25.2 & 33.3 & 21.7 & 35.7 & 22.9 & 37.3 & 29.5 & 30.5 & 18.0 \\
\hline Hungary & 20.0 & 26.6 & 21.1 & 29.5 & 18.2 & 20.4 & 29.0 & 30.4 & 31.2 & 23.4 & 21.5 & 35.6 \\
\hline Poland & 28.4 & 23.1 & 25.9 & 20.6 & 33.2 & 28.8 & 23.9 & 21.5 & 22.4 & 23.7 & 28.9 & 19.9 \\
\hline Romania & 8.5 & 11.2 & 9.3 & 9.9 & 7.1 & 14.0 & 4.5 & 9.4 & 4.1 & 12.4 & 5.8 & 7.2 \\
\hline Slovakia & 7.6 & 15.0 & 7.3 & 14.9 & 8.2 & 15.1 & 6.9 & 15.8 & 5.0 & 11.0 & 13.4 & 19.4 \\
\hline \multicolumn{13}{|c|}{ (c) Transport equipment sector } \\
\hline \multicolumn{13}{|c|}{ Total value (millions US\$) } \\
\hline CEE5 & 5,412 & 45,458 & 1,831 & 22,380 & 3,581 & 23,078 & 6,052 & 78,875 & 1,570 & 29,290 & 4,482 & 49,585 \\
\hline \multicolumn{13}{|c|}{ Share in CEE5 (total value $=100$ ) } \\
\hline Czech & 32.3 & 22.5 & 29.9 & 27.3 & 33.5 & 17.9 & 33.3 & 30.2 & 45.0 & 33.3 & 29.2 & 28.5 \\
\hline Hungary & 19.9 & 11.4 & 11.6 & 13.1 & 24.2 & 9.7 & 11.5 & 11.1 & 21.9 & 14.2 & 7.9 & 9.3 \\
\hline Poland & 30.6 & 39.7 & 46.4 & 27.6 & 22.5 & 51.5 & 38.2 & 31.8 & 11.8 & 30.2 & 47.3 & 32.7 \\
\hline Romania & 7.3 & 9.7 & 4.4 & 9.5 & 8.8 & 10.0 & 7.1 & 9.6 & 5.4 & 9.9 & 7.6 & 9.4 \\
\hline Slovakia & 9.9 & 16.6 & 7.8 & 22.6 & 10.9 & 10.8 & 9.9 & 17.3 & 15.9 & 12.4 & 7.9 & 20.2 \\
\hline
\end{tabular}

(Source) authors' calculation, using data available from UN comtrade.

Table 2. Machinery trade in CEE by origin and destination

\section{(i) CEE}

\begin{tabular}{|c|c|c|c|c|c|c|c|c|}
\hline & \multirow[b]{2}{*}{ Year } & \multirow{2}{*}{$\begin{array}{l}\text { Ori./ } \\
\text { Dest. }\end{array}$} & \multicolumn{3}{|c|}{ Imports } & \multicolumn{3}{|c|}{ Exports } \\
\hline & & & Total & Parts & Final & Total & Parts & Final \\
\hline \multicolumn{9}{|c|}{ (a) All machinery sectors } \\
\hline \multirow{2}{*}{$\begin{array}{l}\begin{array}{l}\text { Value } \\
\text { (millions US\$) }\end{array} \\
\end{array}$} & 1995 & World & 30,005 & 12,730 & 17,275 & 18,064 & 9,659 & 8,405 \\
\hline & 2010 & World & 216,980 & 131,300 & 85,680 & 256,220 & 113,500 & 142,720 \\
\hline \multirow{4}{*}{$\begin{array}{l}\text { Value index } \\
(1995=1)\end{array}$} & 2010 & World & 7.2 & 10.3 & 5.0 & 14.2 & 11.8 & 17.0 \\
\hline & 2010 & Asia & 26.3 & 55.9 & 13.2 & 15.7 & 15.8 & 15.6 \\
\hline & 2010 & WE & 4.6 & 6.8 & 2.9 & 13.8 & 10.8 & 18.5 \\
\hline & 2010 & CEE & 11.8 & 11.5 & 12.2 & 13.4 & 13.3 & 13.5 \\
\hline \multirow{6}{*}{$\begin{array}{l}\text { Share } \\
(\text { in total }(\%) \text { ) }\end{array}$} & 1995 & Asia & 8.7 & 6.3 & 10.4 & 2.9 & 2.7 & 3.3 \\
\hline & 1995 & WE & 59.0 & 61.8 & 56.9 & 51.6 & 58.6 & 43.6 \\
\hline & 1995 & CEE & 6.5 & 8.7 & 4.8 & 12.8 & 12.6 & 13.1 \\
\hline & 2010 & Asia & 31.5 & 33.9 & 27.9 & 3.3 & 3.6 & 3.0 \\
\hline & 2010 & WE & 37.7 & 40.5 & 33.5 & 50.3 & 53.8 & 47.5 \\
\hline & 2010 & CEE & 10.5 & 9.6 & 11.9 & 12.1 & 14.2 & 10.4 \\
\hline \multicolumn{9}{|c|}{ (b) Electric machinery sector } \\
\hline Value & 1995 & World & 8,076 & 5,218 & 2,858 & 5,608 & 4,293 & 1,315 \\
\hline (millions US\$) & 2010 & World & 91,080 & 63,150 & 27,930 & 97,955 & 41,730 & 56,225 \\
\hline
\end{tabular}




\begin{tabular}{|c|c|c|c|c|c|c|c|}
\hline \multirow{4}{*}{$\begin{array}{l}\text { Value index } \\
(1995=1)\end{array}$} & 2010 World & 11.3 & 12.1 & 9.8 & 17.5 & 9.7 & 42.9 \\
\hline & 2010 Asia & 38.8 & 56.9 & 21.3 & 17.5 & 10.7 & 45.5 \\
\hline & $2010 \mathrm{WE}$ & 5.4 & 5.9 & 4.2 & 13.5 & 7.4 & 41.3 \\
\hline & 2010 CEE & 24.5 & 18.5 & 36.1 & 29.8 & 23.7 & 40.0 \\
\hline \multirow{6}{*}{$\begin{array}{l}\text { Share } \\
\text { (in total }(\%) \text { ) }\end{array}$} & 1995 Asia & 12.6 & 9.5 & 18.1 & 2.5 & 2.7 & 2.1 \\
\hline & $1995 \mathrm{WE}$ & 55.8 & 61.4 & 45.6 & 63.2 & 67.8 & 48.5 \\
\hline & 1995 CEE & 5.3 & 5.4 & 5.1 & 8.9 & 7.3 & 14.1 \\
\hline & 2010 Asia & 43.2 & 44.9 & 39.5 & 2.5 & 2.9 & 2.3 \\
\hline & $2010 \mathrm{WE}$ & 26.8 & 29.9 & 19.8 & 48.9 & 51.7 & 46.7 \\
\hline & $2010 \mathrm{CEE}$ & 11.5 & 8.3 & 18.7 & 15.1 & 17.8 & 13.2 \\
\hline \multicolumn{8}{|c|}{ Transport equipment sector } \\
\hline \multirow{2}{*}{$\begin{array}{l}\text { Value } \\
\text { (millions US\$) }\end{array}$} & 1995 World & 5,412 & 1,831 & 3,581 & 6,052 & 1,570 & 4,482 \\
\hline & 2010 World & 45,458 & 22,380 & 23,078 & 78,875 & 29,290 & 49,585 \\
\hline \multirow{4}{*}{$\begin{array}{l}\text { Value index } \\
(1995=1)\end{array}$} & 2010 World & 8.4 & 12.2 & 6.4 & 13.0 & 18.7 & 11.1 \\
\hline & 2010 Asia & 7.6 & 45.3 & 3.4 & 14.1 & 28.9 & 11.8 \\
\hline & $2010 \mathrm{WE}$ & 7.7 & 12.4 & 5.2 & 14.4 & 21.4 & 11.7 \\
\hline & $2010 \mathrm{CEE}$ & 10.0 & 10.0 & 9.9 & 10.3 & 11.3 & 9.6 \\
\hline \multirow{6}{*}{$\begin{array}{l}\text { Share } \\
\text { (in total }(\%) \text { ) }\end{array}$} & 1995 Asia & 10.0 & 3.0 & 13.7 & 2.8 & 1.4 & 3.3 \\
\hline & $1995 \mathrm{WE}$ & 57.5 & 59.4 & 56.5 & 47.2 & 51.1 & 45.8 \\
\hline & $1995 \mathrm{CEE}$ & 11.1 & 18.5 & 7.3 & 12.8 & 22.7 & 9.4 \\
\hline & 2010 Asia & 9.0 & 11.0 & 7.1 & 3.0 & 2.2 & 3.5 \\
\hline & $2010 \mathrm{WE}$ & 52.8 & 60.4 & 45.5 & 52.2 & 58.5 & 48.5 \\
\hline & 2010 CEE & 13.1 & 15.2 & 11.1 & 10.2 & 13.7 & 8.1 \\
\hline
\end{tabular}

(ii) Czech

\begin{tabular}{|c|c|c|c|c|c|c|c|c|}
\hline & \multirow[b]{2}{*}{ Year } & \multirow{2}{*}{$\begin{array}{l}\text { Ori./ } \\
\text { Dest. }\end{array}$} & \multicolumn{3}{|c|}{ Imports } & \multicolumn{3}{|c|}{ Exports } \\
\hline & & & Total & Parts & Final & Total & Parts & Final \\
\hline \multicolumn{9}{|c|}{ (a) All machinery sectors } \\
\hline \multirow{2}{*}{$\begin{array}{l}\text { Value } \\
\text { (millions US\$) }\end{array}$} & 1995 & World & 10,090 & 4,380 & 5,710 & 6,725 & 3,950 & 2,775 \\
\hline & 2010 & World & 56,670 & 35,000 & 21,670 & 73,740 & 34,600 & 39,140 \\
\hline \multirow{4}{*}{$\begin{array}{l}\text { Value index } \\
(1995=1)\end{array}$} & 2010 & World & 5.6 & 8.0 & 3.8 & 11.0 & 8.8 & 14.1 \\
\hline & 2010 & Asia & 30.5 & 58.4 & 18.3 & 6.5 & 9.1 & 3.9 \\
\hline & 2010 & WE & 3.2 & 4.5 & 2.0 & 11.0 & 8.0 & 17.6 \\
\hline & 2010 & CEE & 7.2 & 9.2 & 4.7 & 7.4 & 7.0 & 7.8 \\
\hline \multirow{6}{*}{$\begin{array}{l}\text { Share } \\
\text { (in total }(\%) \text { ) }\end{array}$} & 1995 & Asia & 6.5 & 4.5 & 8.0 & 4.0 & 3.5 & 4.8 \\
\hline & 1995 & WE & 63.6 & 68.3 & 60.0 & 53.9 & 62.9 & 41.3 \\
\hline & 1995 & CEE & 7.7 & 9.9 & 6.0 & 17.5 & 16.0 & 19.6 \\
\hline & 2010 & Asia & 35.1 & 32.9 & 38.6 & 2.4 & 3.6 & 1.3 \\
\hline & 2010 & WE & 36.1 & 38.7 & 31.8 & 54.1 & 57.2 & 51.4 \\
\hline & 2010 & CEE & 9.9 & 11.5 & 7.5 & 11.7 & 12.8 & 10.8 \\
\hline \multicolumn{9}{|c|}{ (b) Electric machinery sector } \\
\hline \multirow{2}{*}{$\begin{array}{l}\text { Value } \\
\text { (millions US\$) }\end{array}$} & 1995 & World & 2,856 & 1,900 & 956 & 2,002 & 1,600 & 402 \\
\hline & 2010 & World & 21,980 & 15,900 & 6,080 & 22,420 & 12,300 & 10,120 \\
\hline \multirow{4}{*}{$\begin{array}{l}\text { Value index } \\
(1995=1)\end{array}$} & 2010 & World & 7.7 & 8.4 & 6.4 & 11.2 & 7.7 & 25.3 \\
\hline & 2010 & Asia & 34.2 & 50.8 & 18.9 & 7.1 & 5.8 & 14.2 \\
\hline & 2010 & WE & 3.5 & 3.6 & 3.4 & 9.6 & 6.2 & 29.6 \\
\hline & 2010 & CEE & 10.3 & 12.2 & 7.9 & 13.0 & 11.1 & 17.0 \\
\hline
\end{tabular}




\begin{tabular}{|c|c|c|c|c|c|c|c|}
\hline \multirow{6}{*}{$\begin{array}{l}\text { Share } \\
\text { (in total }(\%) \text { ) }\end{array}$} & 1995 Asia & 9.3 & 6.7 & 14.5 & 4.8 & 5.0 & 3.9 \\
\hline & $1995 \mathrm{WE}$ & 64.2 & 70.9 & 51.0 & 68.6 & 73.4 & 49.8 \\
\hline & $1995 \mathrm{CEE}$ & 6.7 & 5.7 & 8.8 & 10.9 & 9.3 & 17.3 \\
\hline & 2010 Asia & 41.4 & 40.8 & 43.1 & 3.0 & 3.7 & 2.2 \\
\hline & $2010 \mathrm{WE}$ & 29.5 & 30.4 & 27.2 & 58.8 & 59.3 & 58.3 \\
\hline & $2010 \mathrm{CEE}$ & 9.0 & 8.3 & 11.0 & 12.6 & 13.4 & 11.6 \\
\hline \multicolumn{8}{|c|}{ Transport equipment sector } \\
\hline \multirow{2}{*}{$\begin{array}{l}\text { Value } \\
\text { (millions US\$) }\end{array}$} & 1995 World & 1,746 & 547 & 1,199 & 2,015 & 707 & 1,308 \\
\hline & 2010 World & 10,250 & 6,110 & 4,140 & 23,810 & 9,760 & 14,050 \\
\hline \multirow{4}{*}{$\begin{array}{l}\text { Value index } \\
(1995=1)\end{array}$} & 2010 World & 5.9 & 11.2 & 3.5 & 11.8 & 13.8 & 10.8 \\
\hline & 2010 Asia & 7.2 & 57.6 & 2.1 & 2.4 & 9.8 & 0.2 \\
\hline & $2010 \mathrm{WE}$ & 5.0 & 11.4 & 2.5 & 14.3 & 15.3 & 13.5 \\
\hline & $2010 \mathrm{CEE}$ & 6.5 & 6.7 & 6.0 & 7.9 & 8.5 & 7.4 \\
\hline \multirow{6}{*}{$\begin{array}{l}\text { Share } \\
\text { (in total }(\%) \text { ) }\end{array}$} & 1995 Asia & 7.4 & 2.2 & 9.7 & 4.2 & 2.7 & 5.0 \\
\hline & $1995 \mathrm{WE}$ & 64.6 & 59.6 & 66.8 & 43.3 & 52.0 & 38.5 \\
\hline & $1995 \mathrm{CEE}$ & 12.2 & 27.2 & 5.4 & 21.1 & 27.2 & 17.8 \\
\hline & 2010 Asia & 9.0 & 11.2 & 5.8 & 0.8 & 1.9 & 0.1 \\
\hline & $2010 \mathrm{WE}$ & 55.5 & 60.6 & 47.9 & 52.3 & 57.8 & 48.3 \\
\hline & $2010 \mathrm{CEE}$ & 13.6 & 16.4 & 9.3 & 14.2 & 16.8 & 12.3 \\
\hline
\end{tabular}

(iii) Hungary

\begin{tabular}{|c|c|c|c|c|c|c|c|c|}
\hline & \multirow[b]{2}{*}{ Year } & \multirow{2}{*}{$\begin{array}{l}\text { Ori./ } \\
\text { Dest. }\end{array}$} & \multicolumn{3}{|c|}{ Imports } & \multicolumn{3}{|c|}{ Exports } \\
\hline & & & Total & Parts & Final & Total & Parts & Final \\
\hline \multicolumn{9}{|c|}{ (a) All machinery sectors } \\
\hline \multirow{2}{*}{$\begin{array}{l}\text { Value } \\
\text { (millions US\$) }\end{array}$} & 1995 & World & 4,973 & 2,130 & 2,843 & 3,418 & 2,290 & 1,128 \\
\hline & 2010 & World & 41,640 & 29,700 & 11,940 & 57,000 & 24,800 & 32,200 \\
\hline \multirow{4}{*}{$\begin{array}{l}\text { Value index } \\
(1995=1)\end{array}$} & 2010 & World & 8.4 & 13.9 & 4.2 & 16.7 & 10.8 & 28.5 \\
\hline & 2010 & Asia & 27.6 & 71.9 & 9.5 & 64.0 & 53.6 & 77.5 \\
\hline & 2010 & WE & 5.6 & 8.9 & 2.6 & 13.3 & 8.7 & 28.7 \\
\hline & 2010 & CEE & 28.7 & 35.7 & 22.7 & 43.8 & 63.0 & 33.4 \\
\hline \multirow{6}{*}{$\begin{array}{l}\text { Share } \\
\text { (in total }(\%) \text { ) }\end{array}$} & 1995 & Asia & 9.4 & 6.3 & 11.6 & 1.4 & 1.2 & 1.9 \\
\hline & 1995 & WE & 59.8 & 67.4 & 54.3 & 60.0 & 69.0 & 41.8 \\
\hline & 1995 & CEE & 2.7 & 2.9 & 2.5 & 5.0 & 2.6 & 9.8 \\
\hline & 2010 & Asia & 30.8 & 32.7 & 26.2 & 5.5 & 6.0 & 5.1 \\
\hline & 2010 & WE & 40.3 & 43.0 & 34.1 & 47.8 & 55.1 & 42.0 \\
\hline & 2010 & CEE & 9.2 & 7.5 & 13.5 & 13.2 & 15.3 & 11.5 \\
\hline \multicolumn{9}{|c|}{ (b) Electric machinery sector } \\
\hline \multirow{2}{*}{$\begin{array}{l}\text { Value } \\
\text { (millions US\$) }\end{array}$} & 1995 & World & 1,618 & 1,100 & 518 & 1,624 & 1,340 & 284 \\
\hline & 2010 & World & 24,250 & 18,600 & 5,650 & 29,790 & 9,750 & 20,040 \\
\hline \multirow{4}{*}{$\begin{array}{l}\text { Value index } \\
(1995=1)\end{array}$} & 2010 & World & 15.0 & 16.9 & 10.9 & 18.3 & 7.3 & 70.9 \\
\hline & 2010 & Asia & 68.4 & 112.6 & 28.2 & 71.3 & 22.6 & 841.6 \\
\hline & 2010 & WE & 7.1 & 8.0 & 4.1 & 10.6 & 4.8 & 44.8 \\
\hline & 2010 & CEE & 72.3 & 47.9 & 148.4 & 148.1 & 79.3 & 466.1 \\
\hline \multirow{6}{*}{$\begin{array}{l}\text { Share } \\
\text { (in total }(\%) \text { ) }\end{array}$} & 1995 & Asia & 9.8 & 6.9 & 16.0 & 1.1 & 1.3 & 0.4 \\
\hline & 1995 & WE & 59.1 & 66.1 & 43.8 & 72.5 & 75.0 & 61.1 \\
\hline & 1995 & CEE & 1.8 & 2.0 & 1.3 & 1.8 & 1.8 & 1.9 \\
\hline & 2010 & Asia & 44.9 & 45.8 & 41.3 & 4.4 & 4.0 & 4.6 \\
\hline & 2010 & WE & 28.0 & 31.4 & 16.6 & 42.0 & 49.2 & 38.6 \\
\hline & 2010 & CEE & 8.6 & 5.6 & 18.2 & 14.7 & 19.8 & 12.3 \\
\hline
\end{tabular}


(c) Transport equipment sector

\begin{tabular}{llrrrrrr} 
Value & 1995 World & 1,079 & 212 & 867 & 698 & 343 & 355 \\
(millions US\$) & 2010 World & 5,182 & 2,930 & 2,252 & 8,743 & 4,160 & 4,583 \\
\hline Value index & 2010 World & 4.8 & 13.8 & 2.6 & 12.5 & 12.1 & 12.9 \\
$(1995=1)$ & 2010 Asia & 1.3 & 9.0 & 0.3 & 120.3 & 76.8 & 1436.7 \\
& 2010 WE & 6.2 & 14.2 & 3.5 & 21.4 & 14.2 & 45.2 \\
& 2010 CEE & 13.9 & 28.9 & 7.1 & 15.1 & 42.9 & 10.4 \\
\hline Share & 1995 Asia & 14.7 & 9.0 & 16.1 & 0.4 & 0.7 & 0.0 \\
(in total $(\%))$ & 1995 WE & 52.6 & 67.8 & 48.9 & 33.3 & 52.1 & 15.1 \\
& 1995 CEE & 4.5 & 7.2 & 3.8 & 10.7 & 3.2 & 17.8 \\
& 2010 Asia & 4.1 & 5.8 & 1.8 & 3.4 & 4.4 & 2.5 \\
& 2010 WE & 67.6 & 69.6 & 65.0 & 56.8 & 61.0 & 53.0 \\
& $2010 \mathrm{CEE}$ & 13.0 & 15.0 & 10.5 & 12.9 & 11.2 & 14.4 \\
\hline
\end{tabular}

(iv) Poland

\begin{tabular}{|c|c|c|c|c|c|c|c|c|}
\hline & \multirow[b]{2}{*}{ Year } & \multirow{2}{*}{$\begin{array}{l}\text { Ori./ } \\
\text { Dest. }\end{array}$} & \multicolumn{3}{|c|}{ Imports } & \multicolumn{3}{|c|}{ Exports } \\
\hline & & & Total & Parts & Final & Total & Parts & Final \\
\hline \multicolumn{9}{|c|}{ (a) All machinery sectors } \\
\hline \multirow{2}{*}{$\begin{array}{l}\text { Value } \\
\text { (millions US\$) }\end{array}$} & 1995 & World & 9,431 & 4,030 & 5,401 & 5,052 & 2,020 & 3,032 \\
\hline & 2010 & World & 66,180 & 34,200 & 31,980 & 67,870 & 30,200 & 37,670 \\
\hline \multirow{4}{*}{$\begin{array}{l}\text { Value index } \\
(1995=1)\end{array}$} & 2010 & World & 7.0 & 8.5 & 5.9 & 13.4 & 15.0 & 12.4 \\
\hline & 2010 & Asia & 27.1 & 58.0 & 14.2 & 16.4 & 19.0 & 14.7 \\
\hline & 2010 & WE & 4.5 & 5.6 & 3.6 & 12.6 & 14.1 & 11.7 \\
\hline & 2010 & $\mathrm{CEE}$ & 20.9 & 12.9 & 37.8 & 31.1 & 29.7 & 34.2 \\
\hline \multirow{6}{*}{$\begin{array}{l}\text { Share } \\
\text { (in total }(\%) \text { ) }\end{array}$} & 1995 & Asia & 9.3 & 6.4 & 11.5 & 1.9 & 1.9 & 1.9 \\
\hline & 1995 & WE & 58.3 & 58.6 & 58.1 & 53.3 & 51.6 & 54.5 \\
\hline & 1995 & CEE & 2.8 & 4.4 & 1.6 & 4.1 & 7.1 & 2.1 \\
\hline & 2010 & Asia & 35.9 & 43.9 & 27.4 & 2.3 & 2.4 & 2.3 \\
\hline & 2010 & WE & 37.1 & 39.0 & 35.0 & 50.1 & 48.8 & 51.2 \\
\hline & 2010 & CEE & 8.3 & 6.8 & 9.9 & 9.5 & 14.0 & 5.8 \\
\hline \multicolumn{9}{|c|}{ (b) Electric machinery sector } \\
\hline \multirow{2}{*}{$\begin{array}{l}\text { Value } \\
\text { (millions US\$) }\end{array}$} & 1995 & World & 2,297 & 1,350 & 947 & 1,339 & 961 & 378 \\
\hline & 2010 & World & 21,060 & 13,000 & 8,060 & 21,040 & 9,890 & 11,150 \\
\hline \multirow{4}{*}{$\begin{array}{l}\text { Value index } \\
(1995=1)\end{array}$} & 2010 & World & 9.2 & 9.6 & 8.5 & 15.7 & 10.3 & 29.6 \\
\hline & 2010 & Asia & 32.5 & 54.4 & 17.4 & 16.1 & 20.5 & 9.7 \\
\hline & 2010 & WE & 4.0 & 4.1 & 3.8 & 14.8 & 7.8 & 39.2 \\
\hline & 2010 & CEE & 64.2 & 35.1 & 120.5 & 41.3 & 31.1 & 88.7 \\
\hline \multirow{6}{*}{$\begin{array}{l}\text { Share } \\
\text { (in total }(\%) \text { ) }\end{array}$} & 1995 & Asia & 16.1 & 11.2 & 23.0 & 1.4 & 1.1 & 1.9 \\
\hline & 1995 & WE & 48.5 & 52.5 & 42.4 & 50.6 & 54.7 & 40.0 \\
\hline & 1995 & CEE & 1.5 & 1.7 & 1.2 & 5.8 & 6.6 & 3.6 \\
\hline & 2010 & Asia & 57.2 & 63.4 & 47.2 & 1.4 & 2.2 & 0.6 \\
\hline & 2010 & WE & 21.2 & 22.6 & 18.8 & 47.8 & 41.7 & 52.9 \\
\hline & 2010 & CEE & 10.4 & 6.1 & 17.3 & 15.2 & 20.0 & 10.9 \\
\hline \multicolumn{9}{|c|}{ (c) Transport equipment sector } \\
\hline \multirow{2}{*}{$\begin{array}{l}\text { Value } \\
\text { (millions US\$) }\end{array}$} & 1995 & World & 1,656 & 849 & 807 & 2,309 & 185 & 2,124 \\
\hline & 2010 & World & 18,060 & 6,170 & 11,890 & 25,090 & 8,840 & 16,250 \\
\hline \multirow{4}{*}{$\begin{array}{l}\text { Value index } \\
(1995=1)\end{array}$} & 2010 & World & 10.9 & 7.3 & 14.7 & 10.9 & 47.8 & 7.6 \\
\hline & 2010 & Asia & 24.8 & 33.2 & 21.9 & 21.6 & 600.0 & 15.7 \\
\hline & 2010 & WE & 8.2 & 7.7 & 8.7 & 9.2 & 46.3 & 6.1 \\
\hline & 2010 & $\mathrm{CEE}$ & 15.7 & 6.3 & 79.8 & 40.2 & 63.3 & 21.7 \\
\hline
\end{tabular}




\begin{tabular}{llrrrrrr}
\hline Share & 1995 Asia & 4.3 & 2.2 & 6.6 & 1.5 & 0.2 & 1.6 \\
(in total $(\%))$ & $1995 \mathrm{WE}$ & 65.9 & 62.0 & 70.1 & 62.8 & 61.7 & 63.0 \\
& $1995 \mathrm{CEE}$ & 6.2 & 10.6 & 1.6 & 1.7 & 9.3 & 1.0 \\
& $2010 \mathrm{Asia}$ & 9.9 & 9.9 & 9.9 & 3.0 & 2.4 & 3.3 \\
& $2010 \mathrm{WE}$ & 49.6 & 65.7 & 41.2 & 53.4 & 59.8 & 50.1 \\
& $2010 \mathrm{CEE}$ & 8.9 & 9.2 & 8.8 & 6.2 & 12.4 & 2.9 \\
\hline
\end{tabular}

(v) Romania

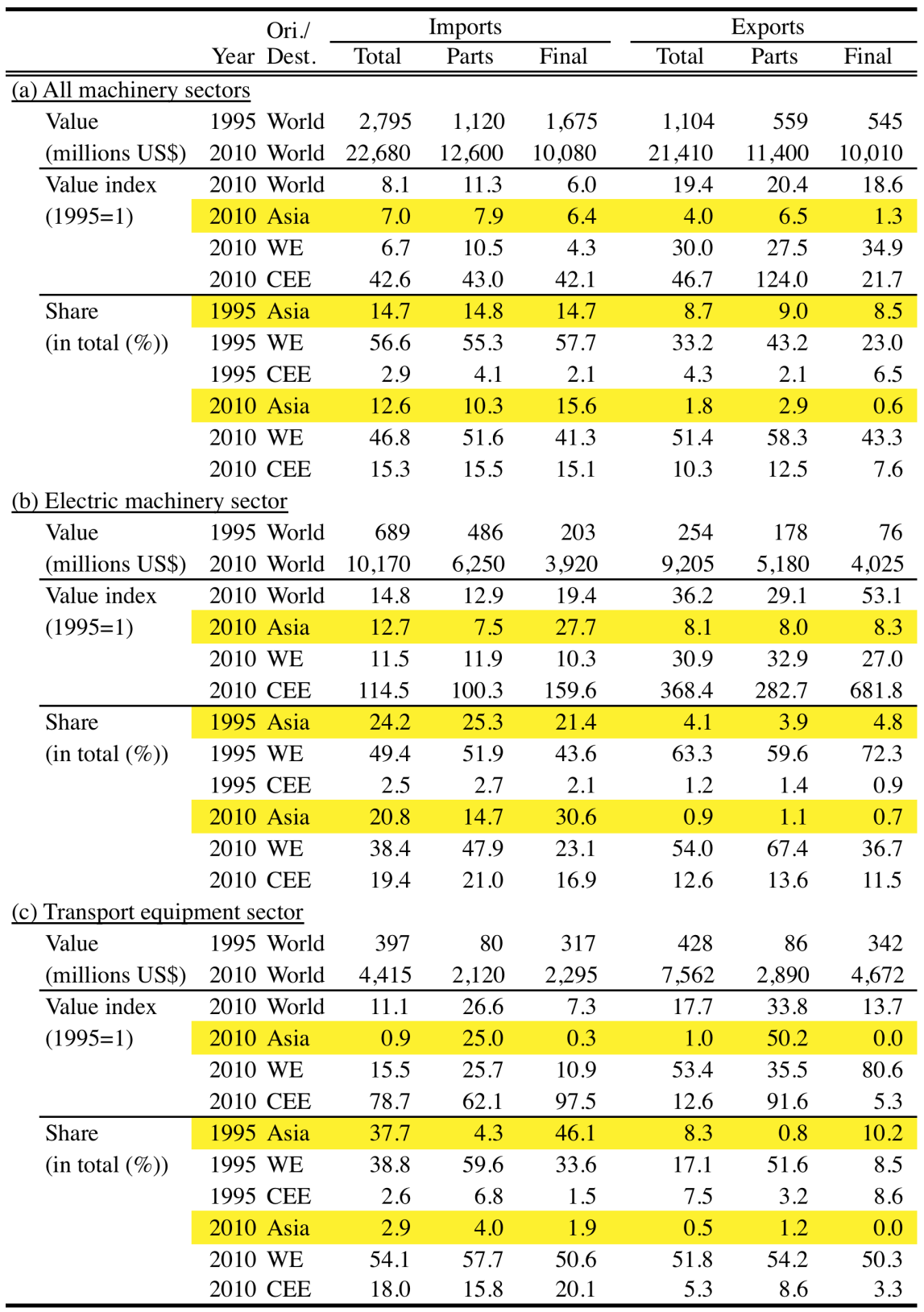


(vi) Slovakia

\begin{tabular}{|c|c|c|c|c|c|c|c|c|}
\hline & \multirow[b]{2}{*}{ Year } & \multirow{2}{*}{$\begin{array}{l}\text { Ori./ } \\
\text { Dest. }\end{array}$} & \multicolumn{3}{|c|}{ Imports } & \multicolumn{3}{|c|}{ Exports } \\
\hline & & & Total & Parts & Final & Total & Parts & Final \\
\hline \multicolumn{9}{|c|}{ (a) All machinery sectors } \\
\hline \multirow{2}{*}{$\begin{array}{l}\text { Value } \\
\text { (millions US\$) }\end{array}$} & 1995 & World & 2,717 & 1,070 & 1,647 & 1,765 & 840 & 925 \\
\hline & 2010 & World & 29,810 & 19,800 & 10,010 & 36,200 & 12,500 & 23,700 \\
\hline \multirow{4}{*}{$\begin{array}{l}\text { Value index } \\
(1995=1)\end{array}$} & 2010 & World & 11.0 & 18.5 & 6.1 & 20.5 & 14.9 & 25.6 \\
\hline & 2010 & Asia & 46.8 & 176.6 & 13.4 & 73.6 & 60.8 & 77.4 \\
\hline & 2010 & WE & 7.7 & 15.4 & 3.1 & 28.4 & 19.6 & 38.8 \\
\hline & 2010 & CEE & 6.5 & 5.7 & 7.6 & 8.7 & 6.1 & 11.5 \\
\hline \multirow{6}{*}{$\begin{array}{l}\text { Share } \\
\text { (in total }(\%) \text { ) }\end{array}$} & 1995 & Asia & 7.1 & 3.7 & 9.3 & 1.1 & 0.6 & 1.7 \\
\hline & 1995 & WE & 44.9 & 42.6 & 46.4 & 33.2 & 37.7 & 29.1 \\
\hline & 1995 & CEE & 25.3 & 35.7 & 18.4 & 40.3 & 43.8 & 37.2 \\
\hline & 2010 & Asia & 30.2 & 35.1 & 20.5 & 4.1 & 2.2 & 5.0 \\
\hline & 2010 & WE & 31.6 & 35.4 & 24.1 & 46.0 & 49.6 & 44.1 \\
\hline & 2010 & CEE & 15.0 & 10.9 & 23.1 & 17.1 & 18.0 & 16.7 \\
\hline \multicolumn{9}{|c|}{ (b) Electric machinery sector } \\
\hline \multirow{2}{*}{$\begin{array}{l}\text { Value } \\
\text { (millions US\$) }\end{array}$} & 1995 & World & 616 & 382 & 234 & 389 & 214 & 175 \\
\hline & 2010 & World & 13,620 & 9,400 & 4,220 & 15,500 & 4,610 & 10,890 \\
\hline \multirow{4}{*}{$\begin{array}{l}\text { Value index } \\
(1995=1)\end{array}$} & 2010 & World & 22.1 & 24.6 & 18.0 & 39.8 & 21.5 & 62.3 \\
\hline & 2010 & Asia & 97.9 & 210.6 & 31.6 & 213.1 & 444.1 & 83.0 \\
\hline & 2010 & WE & 10.4 & 13.4 & 5.2 & 45.5 & 19.0 & 90.5 \\
\hline & 2010 & CEE & 14.4 & 6.6 & 38.4 & 19.2 & 15.8 & 21.8 \\
\hline \multirow{6}{*}{$\begin{array}{l}\text { Share } \\
\text { (in total }(\%) \text { ) }\end{array}$} & 1995 & Asia & 8.6 & 5.2 & 14.3 & 0.2 & 0.1 & 0.2 \\
\hline & 1995 & WE & 43.5 & 44.1 & 42.5 & 40.2 & 46.0 & 33.3 \\
\hline & 1995 & CEE & 25.3 & 30.8 & 16.3 & 43.8 & 34.7 & 55.1 \\
\hline & 2010 & Asia & 38.3 & 44.1 & 25.1 & 0.9 & 2.2 & 0.3 \\
\hline & 2010 & WE & 20.4 & 24.1 & 12.4 & 46.0 & 40.5 & 48.3 \\
\hline & 2010 & CEE & 16.5 & 8.3 & 34.8 & 21.1 & 25.5 & 19.3 \\
\hline \multicolumn{9}{|c|}{ (c) Transport equipment sector } \\
\hline \multirow{2}{*}{$\begin{array}{l}\text { Value } \\
\text { (millions US\$) }\end{array}$} & 1995 & World & 534 & 143 & 391 & 601 & 249 & 352 \\
\hline & 2010 & World & 7,551 & 5,050 & 2,501 & 13,670 & 3,640 & 10,030 \\
\hline \multirow{4}{*}{$\begin{array}{l}\text { Value index } \\
(1995=1)\end{array}$} & 2010 & World & 14.2 & 35.3 & 6.4 & 22.7 & 14.6 & 28.4 \\
\hline & 2010 & Asia & 30.2 & 508.4 & 4.2 & 104.4 & 253.2 & 102.5 \\
\hline & 2010 & WE & 20.7 & 57.1 & 8.0 & 28.0 & 21.7 & 32.7 \\
\hline & 2010 & CEE & 6.7 & 13.3 & 3.0 & 7.6 & 4.2 & 13.8 \\
\hline \multirow{6}{*}{$\begin{array}{l}\text { Share } \\
\text { (in total }(\%) \text { ) }\end{array}$} & 1995 & Asia & 6.6 & 1.3 & 8.5 & 1.7 & 0.1 & 2.9 \\
\hline & 1995 & WE & 31.6 & 30.5 & 32.0 & 38.1 & 39.0 & 37.5 \\
\hline & 1995 & CEE & 42.1 & 56.0 & 37.0 & 34.1 & 53.6 & 20.4 \\
\hline & 2010 & Asia & 14.0 & 18.1 & 5.6 & 7.9 & 0.9 & 10.5 \\
\hline & 2010 & WE & 46.2 & 49.3 & 39.9 & 47.0 & 57.9 & 43.2 \\
\hline & 2010 & $\mathrm{CEE}$ & 19.8 & 21.0 & 17.4 & 11.4 & 15.5 & 9.9 \\
\hline
\end{tabular}

(Source) authors' calculation, using data available from UN comtrade. 
These tables provide several interesting insights. First of all, both machinery imports and exports of CEE5 drastically expanded during the last 15 years. On average, all machinery imports and exports grew by 7 and 14 times in these 15 years, respectively. In particular, imports of machinery parts and components increased by 10 times and exports of machinery final products by 17 times. Combined with the facts that exports of machinery parts and components also increased by 12 times (resulting in the almost same level of import values in 2010) and that major destinations of exports are $\mathrm{WE}$, these figures suggest the development and expansion of production sharing mainly in the WE-CEE nexus.

Second, although WE are still major partners of CEE's machinery trade, CEE's trade with other CEE countries increased in its shares in the electric machinery sector (except exports of final products) and decreased in the transport equipment sector (except imports of final products). The shares of CEE in the electric machinery sector grew from 5 percent to 8 percent for imports in parts and components, 5 percent to 18 percent for imports in final products, and from 7 percent to 18 percent for exports in parts and components. Moreover, both values of imports and exports per se enlarged by more or less 20 times for parts and components and close to 40 times for final products in the electric machinery sector. These figures imply the development of production sharing with expanding fragmentation among CEE countries particularly after the EU enlargement, in addition to the transactions between WE and CEE. ${ }^{11}$

On the other hand, in the transport equipment sector, about 60 percent of imports in parts and components come from WE in both 1995 and 2010. Moreover, export destination is predominantly WE; the export shares of WE increased from 51 percent to 59 percent (46 percent to 49 percent) for parts and components (final products), while the corresponding shares of CEE decreased from 23 percent to 14 percent ( 9 percent to 8 percent). Although the value of trade with other CEE countries in 2010 per se is much larger than that in 1995 even in the transport equipment sector (more or less 10 times of those in 1995), the transactions with WE seem to be getting strengthened.

Third, more interestingly, production networks are not completed within the region particularly in the electric machinery sector in 2010. As mentioned above, transactions among CEE countries became much more active than before. In addition, East Asia plays an extremely important role in this sector from the perspective of production sharing in Europe as well. CEE's imports in machinery goods, mainly parts and components, from East Asia notably increased; in the electric machinery sector, the import value grew by 57 times for parts and components and 21 times for final products during the past 15 years. As a result, the import share of East Asia expanded from 10 percent to 45 percent for parts and components and from 18 percent to 40 percent for final products. ${ }^{12}$ On the other hand, the import share of WE shrunk down

\footnotetext{
'In 2003 before the enlargement of EU, CEE's exports to and imports from other CEE countries remained small (see Ando and Kimura, 2007). At least at that time, international production and distribution networks in Europe did not develop active transactions among CEE countries yet, and the production sharing in Europe was based on the relatively simple WE-CEE nexus.

${ }^{12}$ While the import share of East Asia is high in 2010 for Czech (41 percent for parts and components and 43 percent for final
} 
from 61 percent to 30 percent for parts and components and from 46 percent to 20 percent for final products. These suggest that production networks in this sector obviously expanded from regional to global, with imports of key parts and components as well as finished machinery products from East Asia. As Table A1 in the Appendix clearly shows, many East Asian countries are ranked in the top 20 origins of imports for CEE5 in 2010, particularly in the electric machinery sector; among East Asian countries, China, Korea, and Japan (Korea and Japan) have distinctive high rankings in the electric machinery sector (transport equipment sector).

The import share of East Asia for parts and components in the transport equipment sector is much lower than the corresponding share in the electric machinery sector, though the share increased from 3 percent to 11 percent. $^{13}$ In addition, the export share of East Asia remains small with 1 to 3 percent for both sectors, though the export value expanded by 11 times (29 times) for parts and components and 46 times (12 times) for final products in the electric machinery sector (the transport equipment sector). These also emphasize that production networks expanded from regional to global with a stronger connection with East Asia on the import side in the electric machinery sector, but not so in the transport equipment sector with the exception of Slovakia.

Fourth, the composition of CEE's trade among CEE5 significantly changed. For instance, in the electric machinery sector, Hungary and Slovakia increased in its shares by more than 5 percent for imports in parts and components, while Czech and Poland decreased by more than 5 percent. Moreover, Hungary also increased its shares by more than 10 percent from 22 percent to 36 percent for exports in final products, and Slovakia increased the corresponding share from 13 percent to 19 percent. Furthermore, Slovakia and Romania increased their shares from 5 percent to 11 percent and from 4 percent to 12 percent, respectively, for exports in parts and components, while Czech and Hungary decreased by more than 5 percent. In the transport equipment sector, Slovakia significantly expanded its share while Poland drastically reduced its share for imports in parts and components, and Slovakia significantly expanded its share for exports in final products and Poland for imports of final products. These suggest the development and reshuffling of industrial clusters within forerunners of CEE countries, and Romania has started to be linked with production sharing in other CEE countries.

\footnotetext{
products), Hungary (46 percent and 41 percent), Poland (63 percent and 47 percent), and Slovakia ( 44 percent and 25 percent), the share is not so high for Romania with 15 percent and 31 percent, respectively. Instead, Romania's import (export) share of CEE increased from 3 percent to 21 percent (from 1 percent to 14 percent) for parts and components and from 2 percent to 17 percent (from 1 percent to 12 percent) for final products. These suggest that the direct connection of Romania with East Asia is not so strong but transactions with other CEE countries became much more active for both imports and exports.

${ }^{13}$ For Slovakia, the import share of East Asia for parts and components in the transport equipment sector increased from 1 percent in 1995 to 18 percent in 2010, which is much higher than the corresponding shares for other CEE countries.
} 


\section{B. Features based on the extensive margin}

To further investigate changes in trade patterns or the extent and depth of production networks, this subsection focuses on the extensive margin, that is, the number of traded products and the number of trading partners. Figure 3 demonstrates the number of imported productcountry pairs by three origins in 1995 and 2010 in (a) all machinery sectors (HS 84 to 92), (b) electric machinery sector (HS85), and (c) transport equipment sector (HS 86 to 89) and the corresponding number of exported product-country pairs by three destinations; the number for each CEE country is aggregated as the number for CEE5. The number of product-country pairs for imports from WE and that for imports from East Asia are much greater than those for exports to WE and those to East Asia, while the number for trade with other CEE countries is close to each other. It suggests that CEE countries have a greater variety of product-country pairs of machinery imports from WE and East Asia and export more restricted number of goods, which are produced using imported key parts and components as well as final products, to more restricted destinations.

Figure 3. The number of product-country pairs

Imports

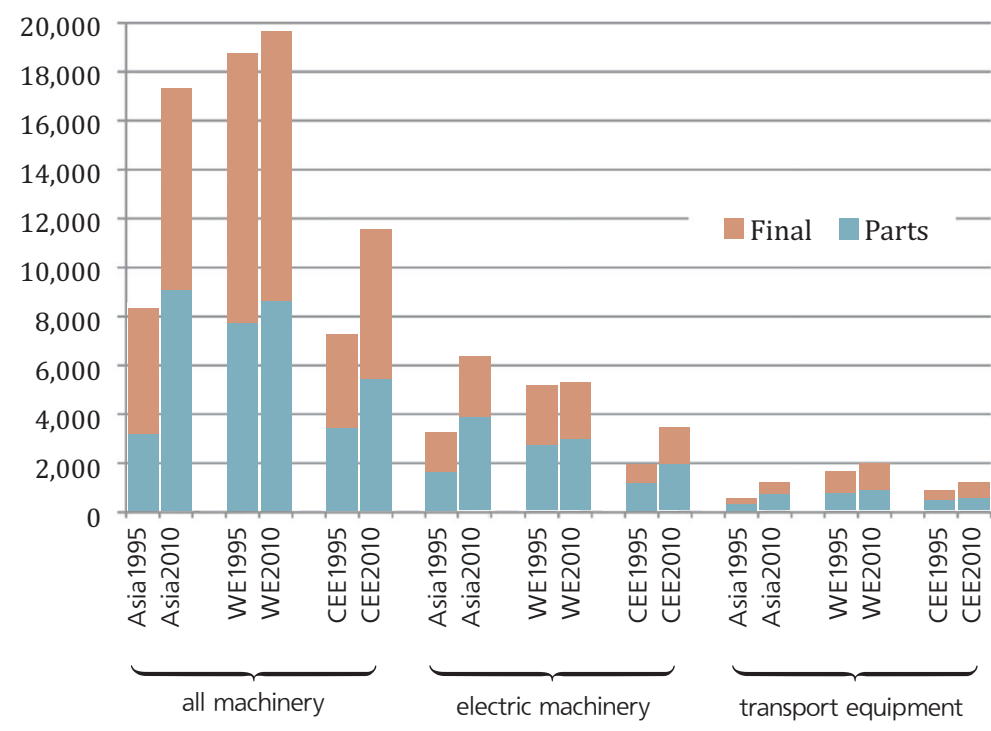




\section{Exports}

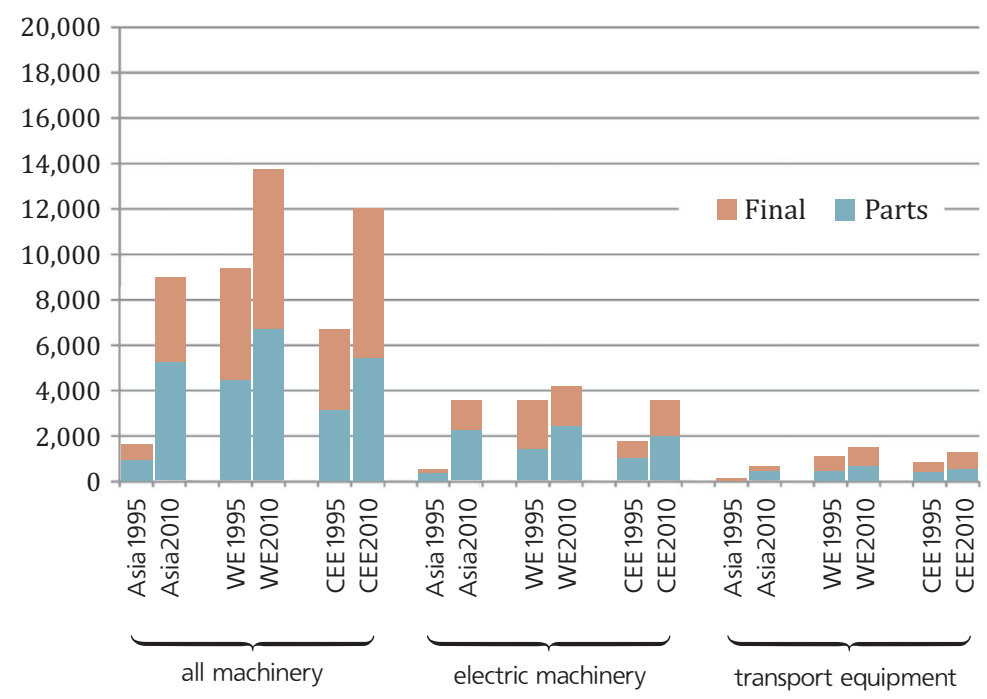

(Source) authors' calculation, using UN comtrade.

Moreover, while the number of product-country pairs of machinery imports from WE does not have a big difference between 1995 and 2010, the corresponding number of imports from East Asia significantly increased by more than twice. As discussed in the previous subsection, values and shares of imports from East Asia drastically expanded. Changes in the extensive margin imply that such an evolution of imports is induced not only by an increase in the intensive margin (increase in the value per product) but also by an increase in the extensive margin (increase in the number of imported product-country pairs).

Furthermore, trade in the electric machinery sector has a greater variety of product-country pairs than that in the transport equipment sector, implying that international transactions in the electric machinery sectors involves more countries and more products.

Figure 4 in turn presents the number of product-country pairs for imports from East Asia, WE, and CEE for each CEE country in 1995, 2000, 2005, and 2010; the number is indexed to the number by three origins for Czech in 1995 because Czech has the largest number for all origins of total machinery trade for both parts and components as well as final products in $1995 .^{14}$

The index smaller than one indicates that the country has been less involved in production networks, compared with the situation of Czech in 1995, and the increasing number of index suggests that the country has been more deeply involved in fragmentation of production than

\footnotetext{
${ }^{14}$ The number of imported product-country pairs for Czech is as follows: 1,956 (total), 896 (parts), and 1,060 (final products) in all machinery sectors; 835, 438, and 397 in the electric machinery sector; and 127, 79, and 48 in the transport equipment sector for East Asia, 4,429, 1,781, and 2,648 in all machinery sectors; 1,178, 624, and 554 in the electric machinery sector; and 395, 162, and 233 in the transport equipment sector for WE, and 2,051, 917, and 1,134 in all machinery sectors; 550, 310, and 240 in the electric machinery sector; and 206, 108, and 98 in the transport equipment sector for CEE.
} 
before. The figure provides three interesting findings. First, all CEE countries dramatically increased in the number of machinery product-country pairs for imports from East Asia, particularly the number for only parts and components, while some countries such as Czech, Hungary, and Slovakia slightly decreased in the corresponding number for imports from WE. ${ }^{15}$ Moreover, all CEE countries increased in the index for CEE in terms of imports as well as exports in the 2000s, indicating that the extent and depth of fragmentation of production enhanced among forerunners of CEE since their EU accession. These figures suggest that transactions of CEE with East Asia become much more active with a greater variety of product-country pairs, with a possible shift of production from WE to East Asia and the development of industrial clusters in CEE mainly after their EU accession, particularly of machinery parts and components.

Figure 4. Number of imported product-country pairs

(a) All machinery sectors

(the number for Czech in 1995=1)

Machinery total

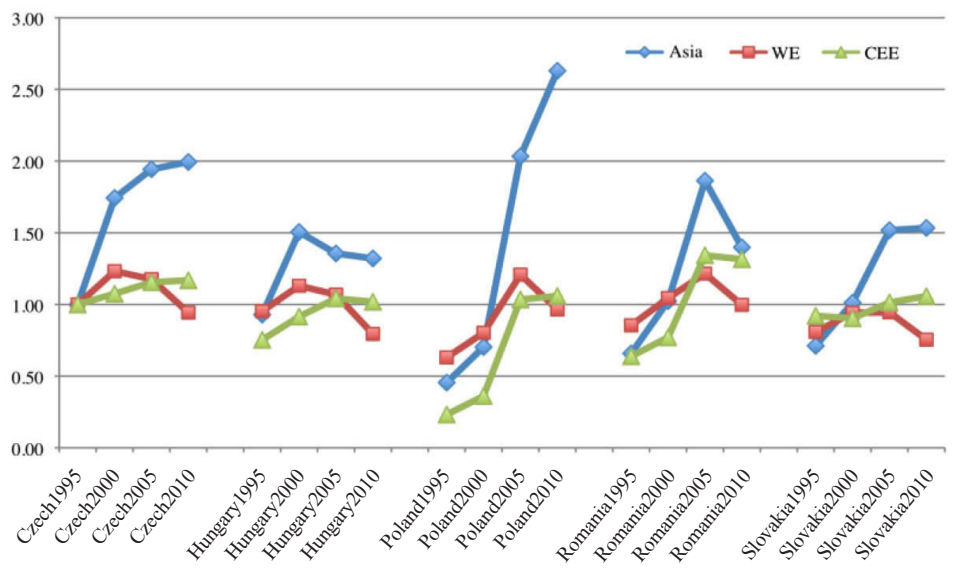

${ }^{15}$ See Figure A1 for the corresponding index for exports. The index for exports also shows expanding connections with East Asia, though the number of product-country pairs for exports to East Asia per se is only a half of that for imports from East Asia in 2010 (Figure 3). 
Machinery parts and components

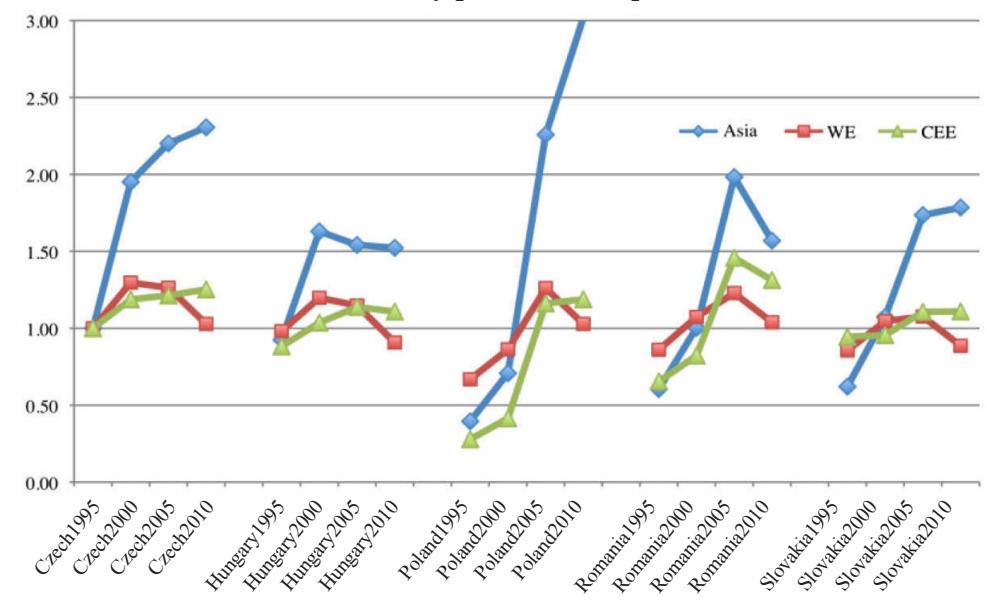

Machinery final products

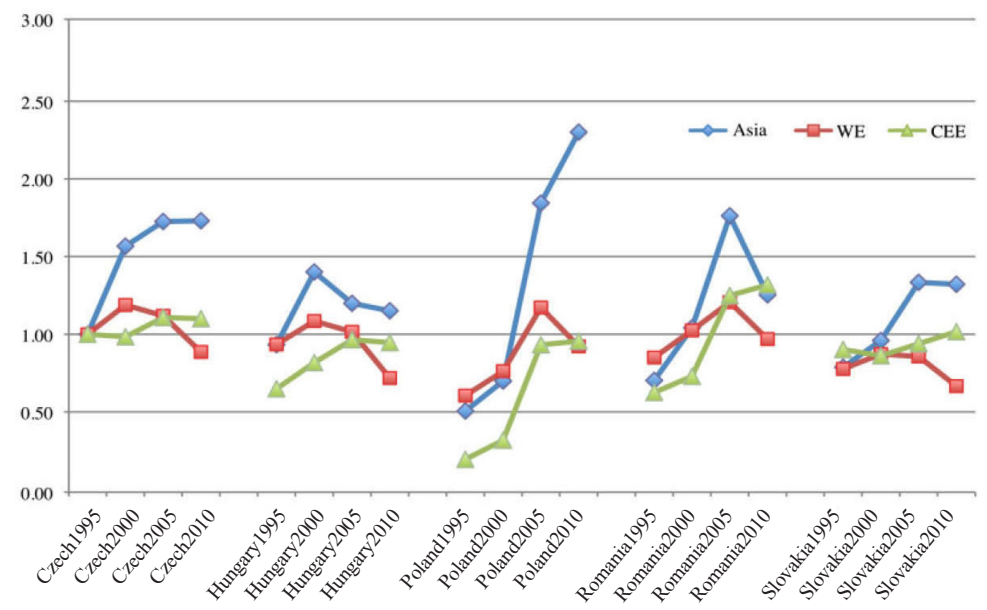

\section{(b) Electric machinery sector}

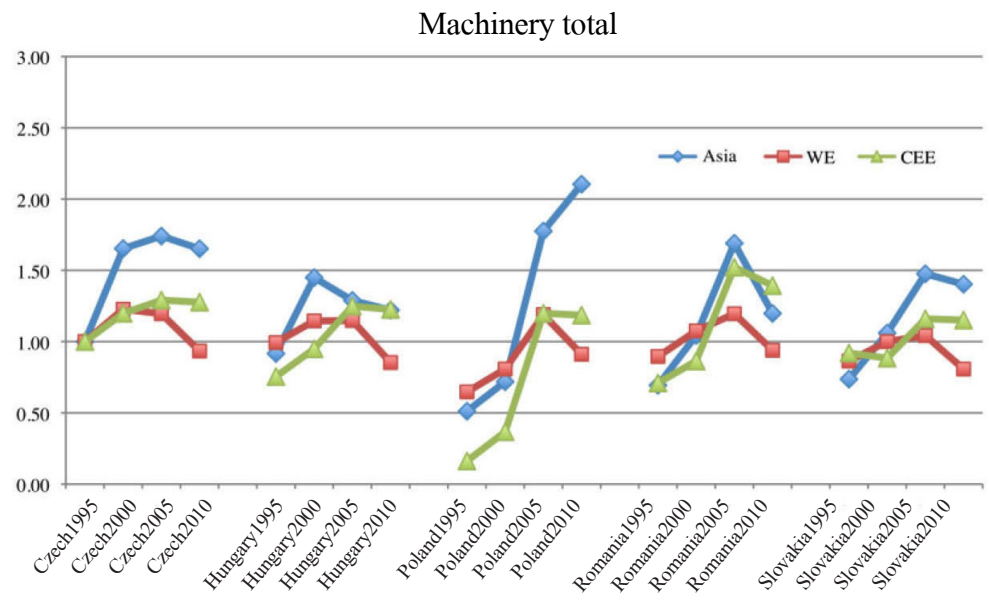


Machinery parts and components

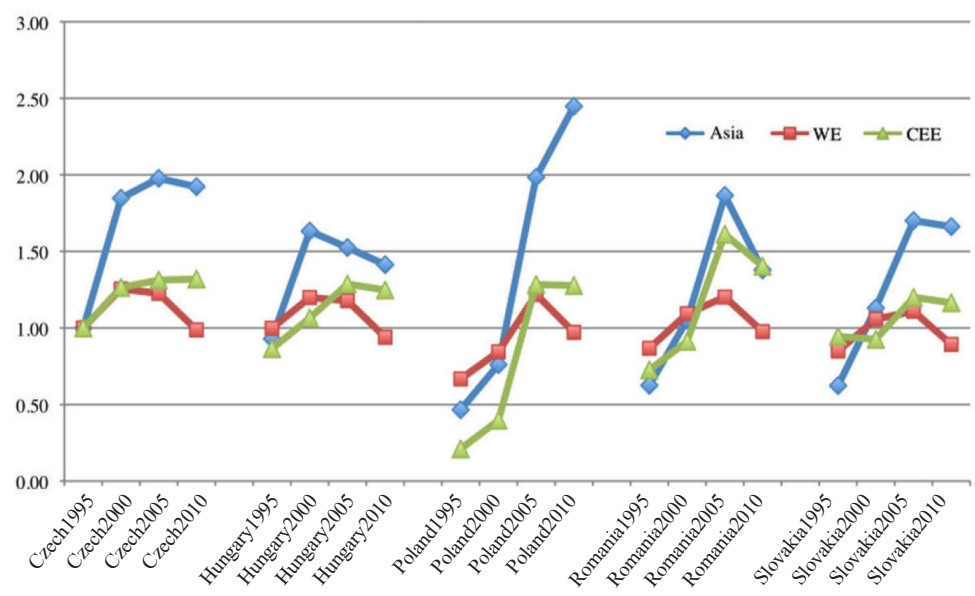

Machinery final products

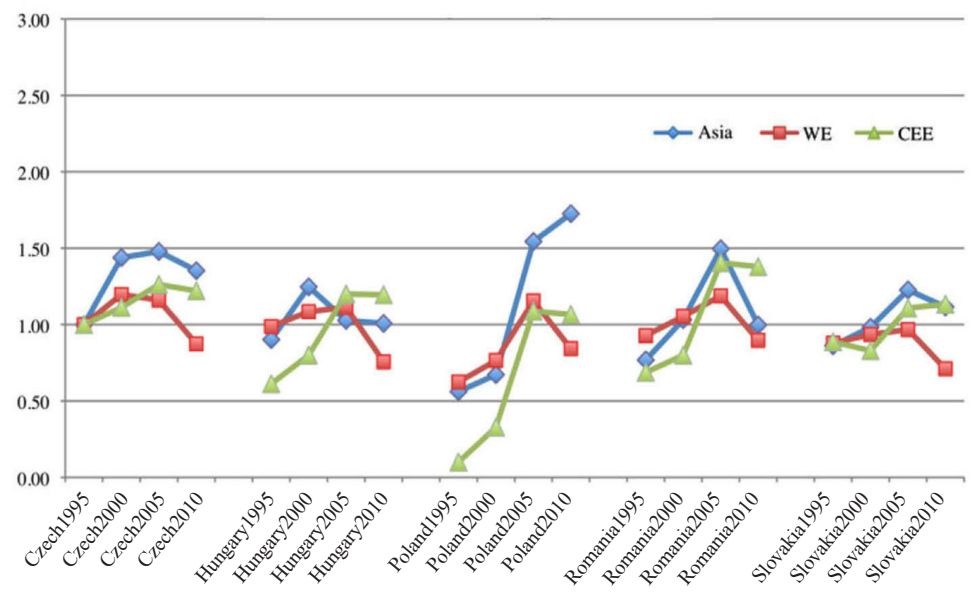

(1

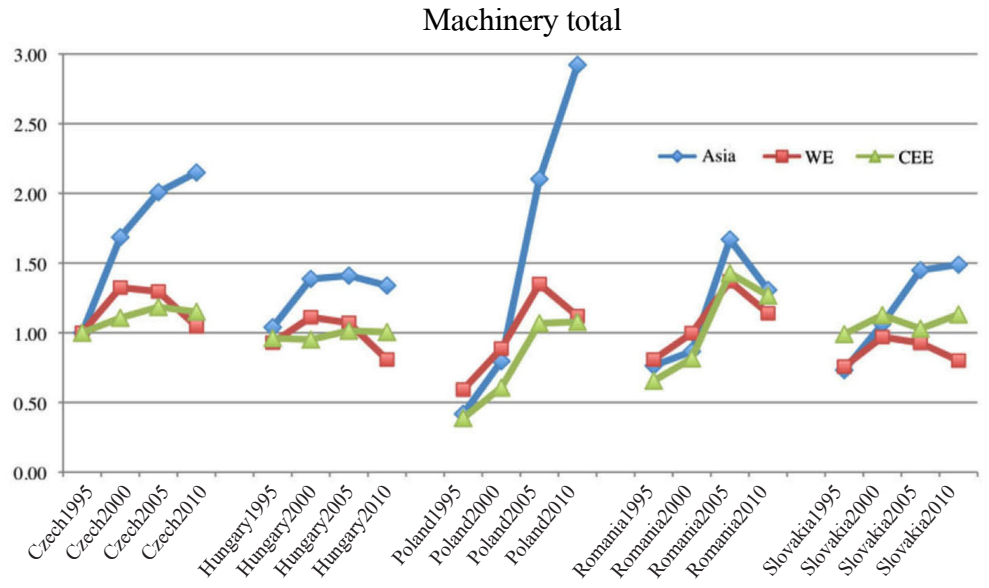


Machinery parts and components

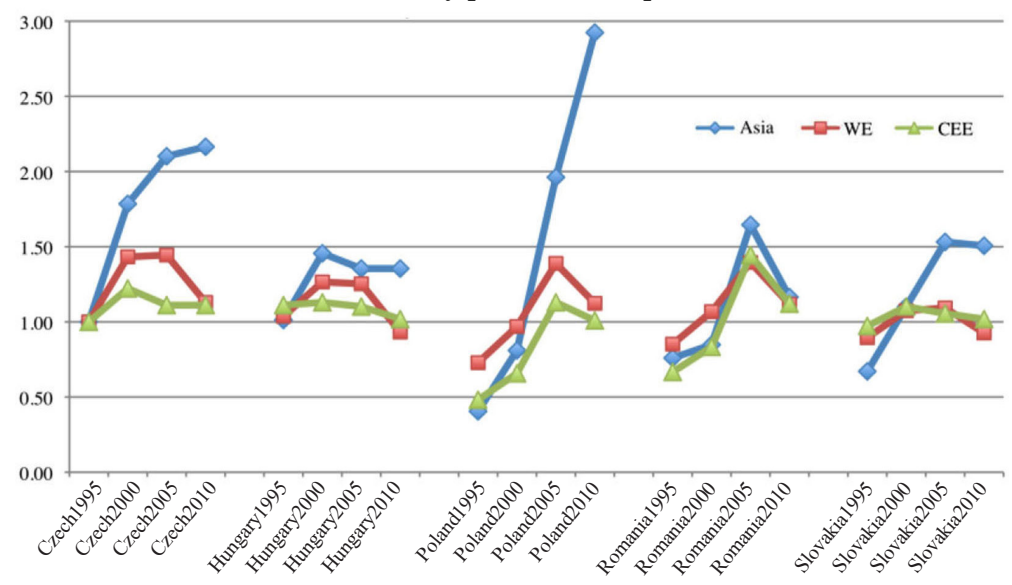

Machinery final products

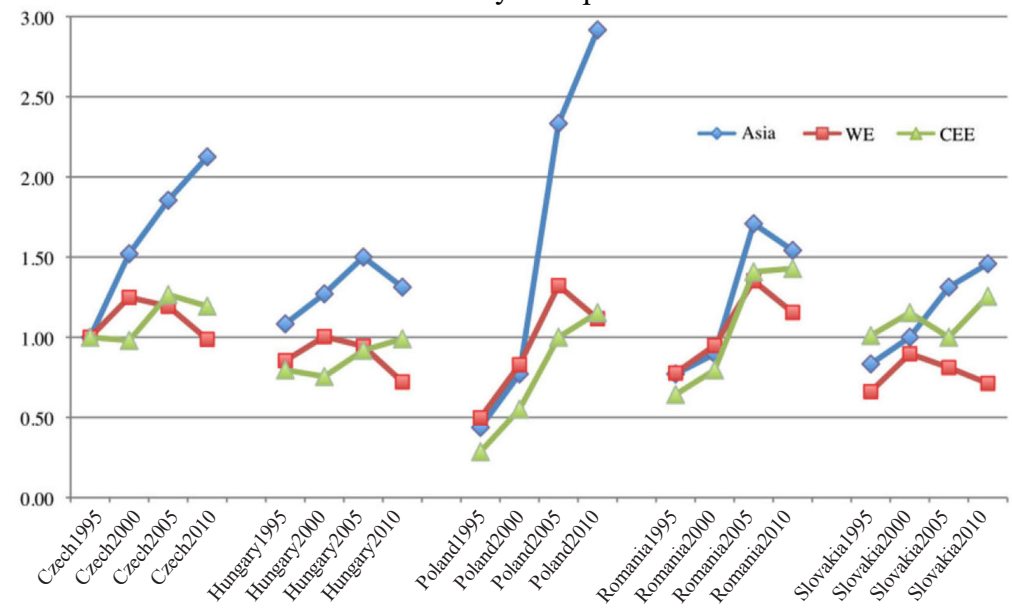

(Source) authors' calculation, using data available from UN comtrade.

Second, the timing of being involved in production networks has been different among CEE countries. According to this index, Czech and Hungary were more advanced in 1995 in terms of the degree of being involved in production networks with active transactions of machinery goods across borders, compared with other CEE countries, particularly in the electric machinery sector. However, Poland, which had the lowest index for all origins in 1995, drastically developed transactions not only with WE but also with East Asia and CEE in the 2000s. As a result, Poland's index for WE and CEE reached almost the same level of indices of Czech and Hungary in the latter half of the 2000s, indicating an active participation of Poland in the production sharing in the 2000s, particularly after the EU accession in 2004. Interestingly, Poland's index for East Asia rapidly increased, particularly for parts and components, and significantly exceeded the corresponding indices of Czech and Hungary. Slovakia also attained higher 
index for East Asia in the 2000s. Moreover, the Romania's index for CEE reached much higher level than those of other CEE countries for both parts and components and final products in the 2000s, with increasing indices for parts and components exports to CEE. Considering the fact that the extent and depth of fragmentation of production has proceeded among forerunners of CEE as mentioned above, Romania seems to start being involved in production networks with a wider range of transactions with other CEE countries, following the development of industrial clusters within forerunners of CEE countries, particularly after joining the EU in 2007.

Third, the connection of CEE with East Asia became stronger in the electric machinery sector than in the transport equipment sector, particularly for imports in parts and components (Figures 3 and 4). Figure 3 clearly shows that the number of product-country pairs for imports in parts and components from East Asia in 2010 is much larger than the corresponding number in 1995 in the electric machinery sector, and also the number in 2010 in the transport equipment sector. In addition, the index for imports in parts and components from East Asia in the electric machinery sector in 2010 tend to be larger than in the transport equipment sector, though the base of the product number is much larger in the former than the latter. ${ }^{16}$ The growth of the extensive margin for imports from East Asia or the connection of CEE with East Asia grew more significantly in the electric sector than the transport equipment sector.

All the descriptive analyses based on the trade value and the extensive margin in this section confirm the growing extent and depth of production networks in Europe from the one based on the simple WE-CEE nexus, to the regional one with more active transactions among CEE countries since their EU accession, and to the global one with a strong connection with East Asia, notably China, Japan, and Korea, particularly in the electric machinery sector.

\section{Machinery Imports of CEE from East Asia}

The previous section descriptively demonstrated expanding fragmentation of production in Europe with a strong connection of CEE with imports from East Asia, based on the total value of trade and the extensive margin. This section quantitatively verifies the existence of such a strong connection with East Asia for machinery imports in CEE and the evolution of production networks from regional to global, using gravity model estimations. The gravity models are estimated for trade in all machinery sectors, trade in the electric machinery sector, and trade in the transport equipment sector, with a distinction between machinery parts, components and final products. Moreover, the paper investigates such patterns not only for the total value of trade but also for the extensive margin (the number of traded product) and the intensive margin (trade value per product) separately. Although exports to East Asia expanded particularly in terms

\footnotetext{
${ }^{16}$ See footnote 14 .
} 
of extensive margins, the export share of East Asia in terms of values are still smaller than the import share of East Asia, and thus this section focuses on a connection of CEE with East Asia from the perspective of imports.

\section{A. Estimation methodology and data}

The basic equation of our gravity model estimations for bilateral machinery imports of CEE5 is as follows:

$$
\ln T_{i j}=\alpha+\beta_{1} \ln D i s t_{i j}+\beta_{2} \ln G D P_{i}+\beta_{3} \ln G D P_{j}+\beta_{4} \ln G D P p c_{i j}+\beta_{5} A s i a+\varepsilon
$$

where $T_{i j}$ denotes the total value of bilateral imports of country $i$ from country $j$, Dist $t_{i j}$ geographical distance between capitals of country $i$ and country $i, G D P_{i}\left(G D P_{j}\right)$ Gross Domestic Products (GDP) of country $i(j), G D P p c_{i j}$ the absolute term of the difference in GDP per capita between country $i$ and country $j$, Asia a dummy variable with one for nine East Asian countries and zero for others. Distance is regarded as a transport cost or services link cost, and the coefficient is supposed to be negative. GDP is a proxy of the market size, and the coefficient is supposed to be positive. The difference in GDP per capita between two countries can be interpreted as a measure of differences in factor endowments. ${ }^{17}$ The coefficient would be positive if the difference in factor endowments is one of the important determinants for the pattern of international division of labor in terms of production processes or tasks as the fragmentation theory suggests. However, now that fragmentation of production becomes networks and trade at the production-process level is active even between developing countries, the difference in factor endowments may not sufficiently capture the overall trade pattern. As for the Asian dummy, the coefficient would be positive if imports from East Asia in terms of the total value as well as extensive and intensive margins are greater than the levels predicted by the model, considering distance and other basic economic conditions.

Since the total value of trade can be rewritten as the trade value per product multiplied by the number of traded product, the total value of trade can be decomposed into the extensive margin (the number of traded product) and the intensive margin (trade value per product) by taking the form of logarithm. Thus, equation (1) can be decomposed into the following two equations:

$$
\ln N_{i j}=\alpha+\beta_{1} \ln D i s t_{i j}+\beta_{2} \ln G D P_{i}+\beta_{3} \ln G D P_{j}+\beta_{4} \ln G D P p c_{i j}+\beta_{5} A s i a+\varepsilon,
$$

\footnotetext{
${ }^{17}$ For instance, Helpman (1987) mentioned that the difference across countries in factor composition can be roughly measured by cross-country differences in income per capita, which actually becomes accurate when there are only two factors of production and all goods are freely traded.
} 


$$
\ln \left(T_{i j} / N_{i j}\right)=\alpha+\beta_{1} \ln D i s t_{i j}+\beta_{2} \ln G D P_{i}+\beta_{3} \ln G D P_{j}+\beta_{4} \ln G D P p c_{i j}+\beta_{5} A s i a+\varepsilon,
$$

where $N_{i j}$ is the number of imported products (extensive margin) and $T_{i j} / N_{i j}$ is the import value per product (intensive margin). This paper regards the number of imported product at the HS 6-digit level as the measure of the extensive margin, namely the number of commodities at the HS 6-digit level with positive import values, and the total values of imports divided by the number of imported product as the measure of intensive margin. ${ }^{1}$

Using ordinary least squares (OLS) regressions based on equations (1) to (3), we investigate the link of CEE with East Asia in both years, 1995 and 2010, for imports in all machinery sectors, those in the electric sector, and those in the transport equipment sector, with a distinction between machinery parts and components and final products. By comparing the results, we would like to capture the features of CEE's imports, particularly those from East Asia and see whether there are any significant changes between 1995 and 2010, between difference machinery sectors, between extenstive and intensive margins, and between parts and components and final products.

Table A2 in the Appendix lists 93 countries in the sample: countries are restricted to those with more than 0.01 percent of machinery trade (imports and/or exports) of CEE5 with the world in 2010 as well as necessary data such as GDP and GDP per capita for both years. The data on trade values in US dollars are obtained from UN comtrade ${ }^{19}$, geographical distance are from the CEPII(Centre d'Etudes Prospectives et d'Informations Internationales)'s database ${ }^{20}$, and GDP and GDP per capita are from the World Development Indicators online ${ }^{21}$. The number of products imported from each country is counted as the number of commodities with positive import values at the HS1992 six-digit level, and the import value per product is obtained by dividing the total value of imports by the number of imported products.

There exist zeros in our bilateral trade matrix. A drop of observations with zero trade cannot utilize potentially useful information and may cause sample selection bias. As suggested in the previous sector, the extensive margin significantly expanded from 1995 to 2010 . For a compar-

\footnotetext{
${ }^{18}$ Our definition of measuring extensive and intensive margins follows Flam and Nordstrom (2011) and Hayakawa, Kimura, and Nabeshima (2011). There are various definitions of extensive and intensive margins for the analysis, using finely disaggregated bilateral trade at the country level (not bilateral trade data at the firm level). For instance, Haddad, Harrison, and Hausman (2010) decompose changes in total trade (the percentage change in the total value of trade) into these margins as follows: intensive margins are composed of effects due to changes in quantity and price; that is, changes in trade values for country-product pairs with positive trade in both periods due to changes in quantity (quantity effect) and changes in price (price effect). Extensive margins consist of an effect due to exiting products (exit effect) and an effect due to new products (entry effect); that is, reduction in trade values due to product-country pairs with positive trade only in the former period and an increase in trade values due to product-country pairs with positive trade only in the latter period. Using this method, Ando and Kimura (2012) emphasize the robustness of transactions of parts and components and the resiliency of the production/distribution networks in East Asia from the perspective of Japanese exports under the Global Financial Crisis and the Great East Japan Earthquake. The examples of other types of definitions include Hummels and Klenow (2005) and Helpman, Melitz, and Rubinstein (2008).

${ }^{19}$ Available from http://comtrade.un.org/db/default.aspx.

${ }^{20}$ Available from http://www.cepii.fr/anglaisgraph/bdd/distances.htm. See Mayer and Zignago (2011) for the details on CEPII's distances measures.

${ }^{21}$ Available from http://databank.worldbank.org/ddp/home.do.
} 
ison of the results in 1995 with those in 2010, it is important to include observations with zero trade, particularly for the estimations on the extensive margin. Therefore, dependent variable (the total value of imports, the number of imported product, or the import value per product) is adjusted by adding one prior to taking the logarithm for the OLS regression analysis.

Our major concerns are the results based on OLS regression analysis as we are interested in the decomposed results into extensive and intensive margins as well. Considering that the treatment of zero-valued trade is regarded as a major issue in the literature, however, the abovementioned gravity equations are also estimated with the Pseudo Poisson Maximum Likelihood (PPML) method, which is proposed by Silva and Tenreyro (2006), for a robustness check. ${ }^{22}$

PPML technique enables us to estimate gravity models, including observations with zero trade, without taking the form of logarithm for dependent variable (that is, the dependent variable is the actual value). Note that equations (2) and (3) do not mean the decomposition of equation (1) any more with PPML technique.

\section{B. The estimation results}

Tables 3 and 4 show the results for the electric machinery sector and the transport equipment sector, respectively. ${ }^{23}$ The major findings are as follows; first, the electric machinery sector is more active in imports from countries with a longer distance than the transport machinery sector is. The coefficient for distance is negative and statistically significant as expected, and the coefficient in the absolute term is smaller for the electric machinery trade than for transport equipment trade in most cases of equations (1) to (3) when the results in the same years are compared. This suggests that the transport equipment sector is more affected by the distance or services link cost than the electric machinery sector. In other words, imports from remote countries are more active in the electric machinery sector and less active in the transport equipment sector. It can be interpreted as a plausible result, probably reflecting the nature of the sector; the transport equipment sector requires industrial clusters nearby as well as higher transport costs, while parts and components in the electric machinery sector, for instance, are in general smaller and more light and thus are relatively easy to be transported countries for a longer distance.

\footnotetext{
${ }^{22}$ Another approach would be the extended technique of the Heckman two-step estimation to take such a systematic sample selection into account; see, for instance, Helpman, Melitz, and Rubinstein (2008).

${ }^{23}$ The results for all machinery sectors are available upon request.
} 


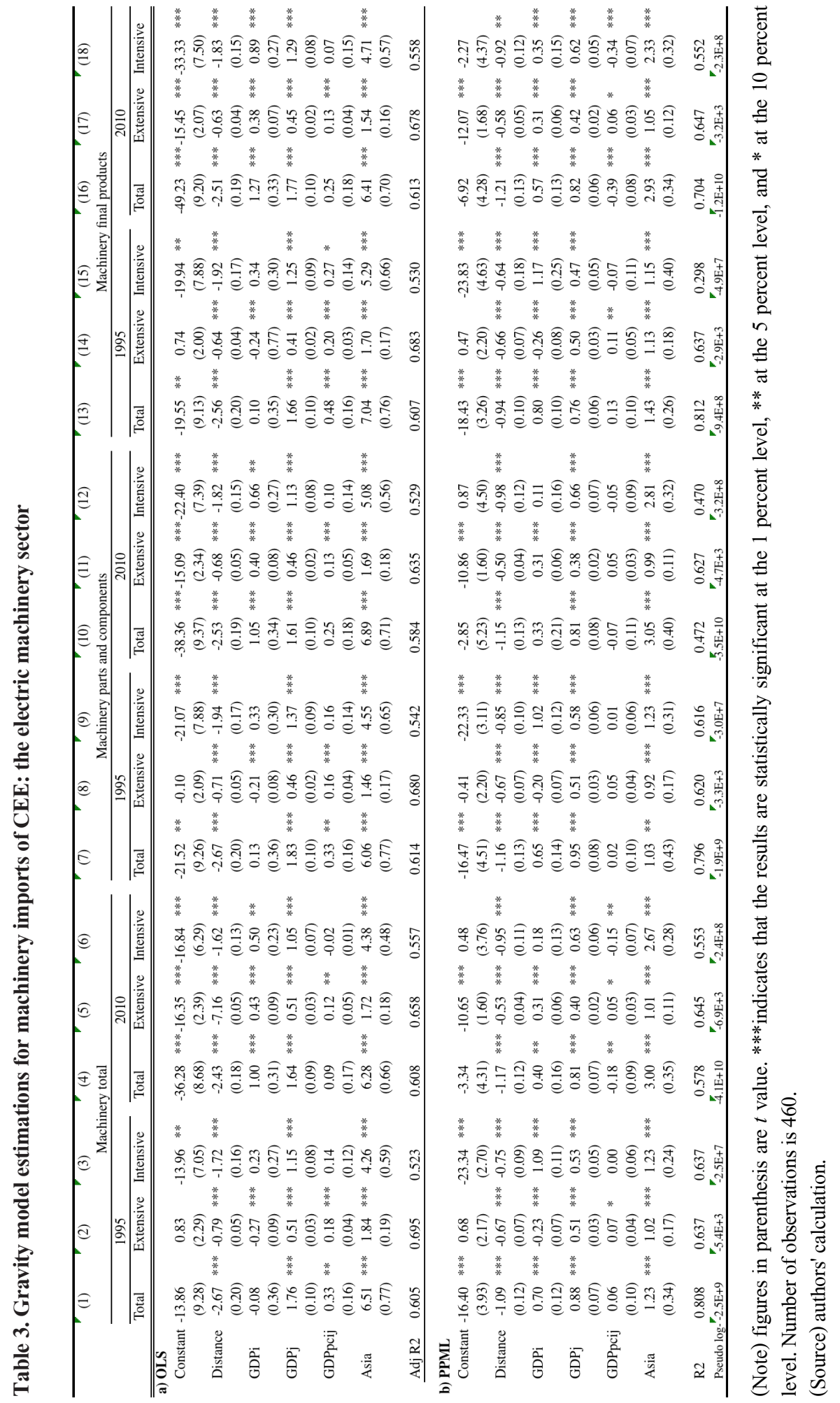




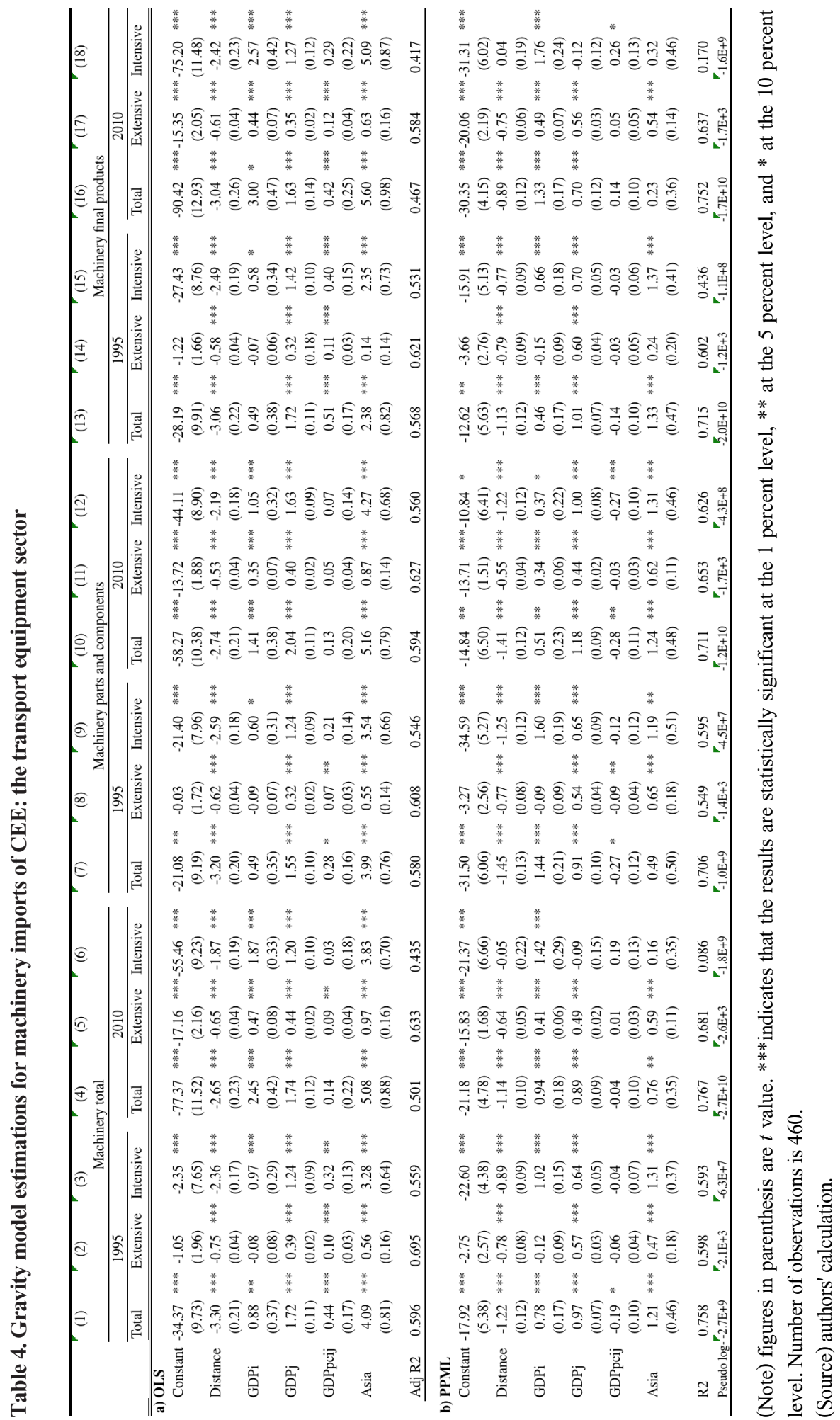


Second, the services link cost is reduced for both sectors. The coefficient for distance in the absolute term becomes smaller in most cases when the results for 1995 are compared with those for 2010. It indicates the reduction in services link cost, which accelerates the international fragmentation of production even beyond the region during the past 15 years.

Third, the CEE's connection with East Asia is strong and becomes tighter, particularly from the perspective of imports in parts and components in the electric machinery sector. The coefficient for East Asia is positive and mostly statistically significant. In addition, the coefficient is much greater for the electric machinery sector than the transport equipment sector when the results in the same years are compared. Moreover, the coefficient became larger particularly for trade in parts and components in both sectors. With a control of distance and other economic conditions, these results suggest that the CEE's connection with East Asia is stronger for both sectors than the average predicted by the model, which is particularly true for the electric machinery sector.

Fourth, CEE's imports from East Asia enlarged not only due to an expansion of the import value per product but also due to an increase in the number of imported products. The abovementioned features, particularly the third point, are observed not only for the total value of imports but also for the number of imported products (extensive margin) as well as the import value per product (intensive margin). It implies that CEE's imports from East Asia grow not only as the results of an expansion of the trade value per product but also as the results of an increase in the number of variety. Following the further development and restructuring of production networks in East Asia involving many countries at different income levels, the production networks in Europe have expanded even beyond the region, in a strong connection with many East Asian countries. ${ }^{24}$

Fifth, the number of imported products is more likely to grow in the electric machinery sector compared with the transport equipment sector, though the imported value per commodity is more likely to expand compared with the number of product in the same sector. The intensive margin tends to be more affected by distance and other variables including the Asian dummy than the extensive margin does for both sectors, but the relative weight of the intensive margin is larger for the transport equipment sector than for the electric machinery sector. For instance, the portion of the intensive margin for the estimations of parts and components and final product is 73 to 75 percent for distance ( 75 to 76 percent for Asia dummy) in the electric machinery sector, and 79 to 80 percent ( 83 to 94 percent) in the transport equipment sector. It suggests that the number of imported commodities is more likely to grow in the electric machinery sector, while the imported value per commodity is more likely to expand in the transport equipment sector.

\footnotetext{
${ }^{24}$ See Ando (2012) for the features of development and restructuring of regional production/distribution networks in East Asia; the paper investigates not only trade patterns of East Asia but also examines the extent and depth of regional production/distribution networks in terms of extensive margins and demonstrate how the networks are being restructured in East Asia, particularly after the Global Financial Crisis.
} 
Regarding control variables other than distance, the coefficient for GDP in exporting country are positive and statistically significant in most estimations, suggesting that CEE tend to import machinery goods from countries with the larger market size such as countries in WE. As for GDP in importing country, the coefficients for the analysis in 2010 are mostly positive and statistically significant but not necessarily statistically significant for the analysis in 1995, implying that CEE countries with the larger market size are likely to export machinery goods, particularly recently. Regarding the difference in GDP per capita, the results are mixed; some are positive and statistically significant while others are not. As previously mentioned, the difference in factor endowments at the macro level may not sufficiently capture the overall trade pattern with fragmentation of production.

\section{Conclusion}

This paper investigated changes in patterns of machinery trade in CEE to analyze the extent and depth of production networks in Europe from the perspective of their links with East Asia via CEE. Our descriptive analysis based on the total value of trade and the extensive margin clearly demonstrated expanding fragmentation of production in Europe with a strong connection of CEE with machinery imports from East Asia. The extent and depth of production networks in Europe grew from the one based on the simple WE-CEE nexus to the regional one with more active transactions among CEE countries mainly after their EU accession, and further to the global one with a strong connection with East Asia, particularly in the electric machinery sector. Our quantitative analysis with not only on the total value of trade but also extensive and intensive margins using gravity models, also verified the existence of such a strong connection with East Asia for machinery imports in CEE and the evolution of production networks in Europe from regional to global. These evidences partially reflect the reduction in services link costs, the evolution of industrial clustering among CEE countries particularly after their joining the EU, and the strengthening competence for production networks in East Asia in machinery sectors. Although it depends on the industries and products with different technologies, service link costs, and business models, our results suggest the possibility of the further development of global production networks if the condition meets.

In order to allow global production networks to develop, it is important to further reduce services link costs to activate production sharing and develop industrial clusters. The reduction in tariffs and non-tariff measures (NTMs) as well as the implementation of various trade and investment facilitation measures should contribute to the reduction in services link costs. One of the ways to carry them out is the utilization of effective free trade agreements (FTAs).

Given the fact that production networks in Europe evolved from the simple WE-CEE nexus to the regional one with active transactions among CEE countries mainly after their EU ac- 
cession, and further to the global one with a strong connection with East Asia, not only FTAs within the same region but also those beyond the region may play a more important role than before in reducing those services link costs. For example, the recently concluded EU-Korea FTA as well as the EU-Japan FTA under review may work for enhancing links between CEE and East Asia.

For FTAs to be effective, the contents of FTAs must be comprehensive, covering not only trade barriers but also various trade and investment facilitation measures. In addition, the tariff structure as well as the rules of origin of FTAs should be constructed so that FTAs are actually utilized. As for industrial clusters, particularly for developing countries, it is important to improve business environment to become a part of industrial clustering and to be involved in production networks. If these improvements are taken into place, the integration between Europe and East Asia and links even with other parts of the world would be further expanded and strengthened.

The paper simply focused on the linkage between Europe and East Asia, but similar connections beyond the geographical region may exist in other links. Although the scale of transactions must be much smaller than the link through CEE, Mexico may playing a bridging role between East Asia and the US. Further analysis to capture the global links from the perspective of production sharing would be worth doing.

Received 12 December 2012, Revised 3 April 2013, Accepted 2 May 2013

\section{References}

Ando, Mitsuyo (2006) "Fragmentation and Vertical Intra-industry Trade in East Asia". North American Journal of Economics and Finance, 17 (3), 257-281.

Ando, Mitsuyo (2012) "Development and Restructuring of Regional Production/Distribution Networks in East Asia”. Prepared for the ERIA Research Project and Presented at International Conference, 'Moving toward a New Development Model for East Asia-The Role of Domestic Policy and Regional Cooperation'.

Ando, Mitsuyo and Fukunari Kimura (2005) “The Formation of International Production and Distribution Networks in East Asia”. In Takatoshi Ito and Andrew Rose, eds., International Trade (NBER-East Asia Seminar on Economics, Volume 14), Chicago: The University of Chicago Press. 177-213.

Ando, Mitsuyo and Fukunari Kimura (2007) "Fragmentation in Europe and East Asia: Evidences from International Trade and FDI Data". In Pierre-Bruno Ruffini and Jong-Kil Kim eds., Corporate Strategies in the Age of Regional Integration: Edward Elgar. 52-76.

Ando, Mitsuyo and Fukunari Kimura (2009) "Fragmentation in East Asia: Further Evidence". ERIA Discussion Paper Series No. 2009-20. A shorter version is appeared with a different title,"The Special Patterns of Production and Distribution Networks in East Asia, " in Prema-chandra Athukorala ed. The Rise of Asia: Trade and Investment in Global Perspective, Routledge in the Growth Economies of Asia. 2010.

Ando, Mitsuyo and Fukunari Kimura (2012) "How Did the Japanese Exports Respond to Two Crises in the 
International Production Network?: The Global Financial Crisis and the Great East Japan Earthquake". Asian Economic Journal, 26 (3), 259-285.

Ando, Mitsuyo, Sven Arndt, and Fukunari Kimura (2009) "Production Networks in East Asia: Strategic Behavior by Japanese and U.S. Firms". Keio Business Review No. 45-2, 21-54.

Arndt, W. Sven, and Henryk Kierzkowski (2001). Fragmentation: New Production Patterns in the World Economy. Oxford: Oxford University Press.

Baldwin (2011) "21st Century Regionalism: Filling the Gap between 21st Century Trade and 20th Century Trade Rules". CEPR Policy Insight No. 56.

Deardorff, Alan. V (2001) "Fragmentation in Simple Trade Models". North American Journal of Economics and Finance, 12. 121-137.

Flam, Harry and Hakan Nordstrom (2011) "Gravity Estimation of the Intensive and Extensive margins of Trade: An Alternative Procedure with Alternative Data". CESifo Working Paper No. 3387.

Haddad, Mona, Ann Harrison, and Catherine Hausman (2010) "Decomposing the Great Trade Collapse: Products, Prices, and Quantities in the 2008-2009 Crisis", NBER Working Paper No. 16253.

Hayakawa, Kazunobu, Fukunari Kimura, and Kaoru Nabeshima (2011) "Non-conventional Provisions in Regional Trade Agreements: Do They Enhance International Trade?" IDE Discussion Paper No. 310 (September).

Helpman, Elhanan (1987), Imperfect Competition and International Trade: Evidence from Fourteen Industrialized Countries, Journal of the Japanese and International Economies, 1, pp. 62-81.

Helpman, Elhanan, Marc Melitz, and Yona Rubinstein (2008). "Estimating Trade Flows: Trading Partners and Trading Volumes". Quarterly Journal of Economics, 123(2), 441-487.

Hummels, David and Peter J. Klenow (2005) “The Variety and Quality of a Nation's Exports". American Economic Review, 95(3), 704-723.

Jones, W. Ronald and Henryk Kierzkowski (1990) "The Role of Services in Production and International Trade: A Theoretical Framework". In Ronald W. Jones and Anne O. Krueger, eds., The Political Economy of International Trade: Essays in Honor of Robert E. Baldwin. Oxford, Basil Blackwell.

Kimura, Fukunari and Mitsuyo Ando (2005) "Two-dimensional Fragmentation in East Asia: Conceptual Framework and Empirics". International Review of Economics and Finance 14, 317-348.

Mayer, Thierry and Soledad Zignago (2011) "Notes on CEPII's distances measures: The GeoDist database". CEPII Working Paper No. 2011-25.

Silva, J.M.C. Santos and Silvana Tenreyro (2006) "The Log of Gravity”, Review of Economics and Statistics, 88(4), 641-658. 
Table A1. The rankings of trade partners and shares in trade with the world

\begin{tabular}{|c|c|c|c|c|c|c|c|c|c|c|c|c|c|c|c|c|c|c|}
\hline & \multicolumn{6}{|c|}{ All machinery sectors } & \multicolumn{6}{|c|}{ Electric machinery sector } & \multicolumn{6}{|c|}{ Transport equipment sector } \\
\hline & \multicolumn{2}{|l|}{ Total } & \multicolumn{2}{|l|}{ Parts } & \multicolumn{2}{|l|}{ Final } & \multicolumn{2}{|l|}{ Total } & \multicolumn{2}{|l|}{ Parts } & \multicolumn{2}{|l|}{ Final } & \multicolumn{2}{|l|}{ Total } & \multicolumn{2}{|l|}{ Parts } & \multicolumn{2}{|l|}{ Final } \\
\hline & Name & $\%$ & Name & $\%$ & Name & $\%$ & Name & $\%$ & Name & $\%$ & Name & $\%$ & Name & $\%$ & Name & $\%$ & Name & $\%$ \\
\hline \multicolumn{19}{|c|}{ Year: 1995} \\
\hline 1 & DEU & 33.1 & DEU & 36.5 & DEU & 30.5 & DEU & 32.5 & DEU & 38.2 & DEU & 22.0 & DEU & 31.6 & DEU & 33.9 & DEU & 30.4 \\
\hline 2 & ITA & 9.5 & ITA & 9.0 & ITA & 9.8 & AUT & 7.0 & AUT & 7.7 & JPN & 7.9 & ITA & 9.5 & ITA & 13.0 & FRA & 10.4 \\
\hline 3 & AUT & 6.2 & AUT & 6.9 & USA & 6.5 & GBR & 6.1 & GBR & 6.1 & FRA & 6.5 & FRA & 8.9 & CZE & 7.8 & ITA & 7.8 \\
\hline 4 & USA & 5.9 & USA & 5.1 & FRA & 5.8 & USA & 5.8 & USA & 5.5 & USA & 6.3 & CZE & 5.7 & SVK & 7.1 & ESP & 6.9 \\
\hline 5 & FRA & 5.4 & FRA & 4.7 & AUT & 5.6 & FRA & 5.7 & FRA & 5.2 & GBR & 6.0 & ESP & 5.0 & SWE & 7.1 & KOR & 6.8 \\
\hline 6 & GBR & 4.9 & GBR & 4.6 & GBR & 5.1 & ITA & 4.6 & ITA & 4.3 & AUT & 5.8 & JPN & 5.0 & FRA & 6.1 & JPN & 6.6 \\
\hline 7 & JPN & 3.9 & CZE & 3.9 & JPN & 5.0 & JPN & 4.3 & KOR & 3.7 & ITA & 5.2 & KOR & 4.6 & AUT & 3.8 & CZE & 4.6 \\
\hline 8 & CZE & 2.9 & SVK & 2.9 & KOR & 2.7 & KOR & 3.4 & NLD & 3.1 & FIN & 4.6 & AUT & 4.1 & GBR & 2.6 & AUT & 4.3 \\
\hline 9 & SWE & 2.7 & SWE & 2.7 & NLD & 2.6 & NLD & 2.9 & CZE & 2.3 & SWE & 3.2 & SWE & 3.8 & JPN & 1.8 & GBR & 3.7 \\
\hline 10 & NLD & 2.6 & NLD & 2.4 & CHE & 2.6 & FIN & 2.5 & JPN & 2.3 & KOR & 2.9 & SVK & 3.6 & HUN & 1.8 & BGD & 2.9 \\
\hline 11 & CHE & 2.4 & JPN & 2.4 & SWE & 2.6 & SWE & 2.5 & SWE & 2.0 & SVK & 2.8 & GVR & 3.3 & POL & 1.7 & RUS & 2.2 \\
\hline 12 & SVK & 2.3 & CHE & 2.1 & CZE & 2.1 & CHE & 2.1 & BGD & 2.0 & CHE & 2.6 & BGD & 2.5 & USA & 1.7 & SWE & 2.2 \\
\hline 13 & KOR & 2.3 & BGD & 1.7 & ESP & 2.1 & CZE & 2.1 & CHE & 1.8 & NLD & 2.5 & NLD & 1.8 & BGD & 1.6 & NLD & 2.1 \\
\hline 14 & BGD & 1.7 & KOR & 1.7 & SVK & 1.9 & SVK & 2.0 & SVK & 1.6 & $\mathrm{CHN}$ & 2.4 & RUS & 1.7 & ESP & 1.4 & SVK & 1.9 \\
\hline 15 & ESP & 1.7 & ESP & 1.1 & BGD & 1.7 & BGD & 1.9 & HKG & 1.5 & BGD & 1.7 & USA & 1.5 & NLD & 1.1 & USA & 1.4 \\
\hline 16 & FIN & 1.3 & POL & 1.1 & DNK & 1.5 & HKG & 1.5 & FIN & 1.4 & CZE & 1.6 & HUN & 0.8 & RUS & 0.7 & CHE & 0.7 \\
\hline 17 & DNK & 1.2 & FIN & 1.1 & FIN & 1.4 & CHN & 1.2 & POL & 1.0 & MYS & 1.6 & POL & 0.8 & DNK & 0.6 & BLR & 0.3 \\
\hline 18 & RUS & 0.7 & DNK & 0.9 & RUS & 0. & ESP & 1.2 & ESP & 1.0 & ESP & 1.5 & CHE & 0.6 & BLR & 0.5 & HUN & 0.3 \\
\hline 19 & SGP & 0.7 & HKG & 0.8 & SGP & 0.8 & SGP & 1.1 & SGP & 0.9 & HKG & 1.5 & BLR & 0.4 & CHE & 0.4 & FIN & 0.3 \\
\hline 20 & POL & 0.7 & HUN & 0.8 & $\mathrm{CHN}$ & 0.8 & & 0.8 & $\mathrm{CHN}$ & 0.6 & SGP & 1.4 & DNK & 0.3 & UKR & 0.4 & POL & 0.3 \\
\hline \multicolumn{19}{|c|}{ Year: 2010} \\
\hline 1 & DEU & 24.2 & DEU & 26.9 & DEU & 20.2 & CHN & 22.3 & CHN & 20.5 & CHN & 26.3 & DEU & 35.3 & DEU & 42.3 & DEU & 28.4 \\
\hline 2 & CHN & 15.1 & CHN & 14.0 & CHN & 16.8 & DEU & 17.6 & DEU & 20.2 & DEU & 11.9 & & 7.3 & FRA & 7.7 & FRA & 7.0 \\
\hline 3 & KOR & 8.0 & KOR & 11.2 & ITA & 4.8 & KOR & 11.1 & KOR & 13.7 & HUN & 7.2 & CZE & 5.9 & CZE & 6.4 & CZE & 5.4 \\
\hline 4 & ITA & 4.8 & ITA & 4.9 & FRA & 3.7 & HUN & 4.2 & NLD & 5.0 & KOR & 5.1 & & 5.5 & ITA & 6.0 & ITA & 5.0 \\
\hline 5 & FRA & 3.9 & JPN & 4.5 & USA & 3.7 & NLD & 4.2 & JPN & 4.0 & SVK & 4.0 & ESP & 4.2 & KOR & 5.5 & ESP & 4.9 \\
\hline 6 & JPN & 3.8 & FRA & 4.1 & NLD & 3.5 & JPN & 3.7 & HUN & 2.9 & POL & 3.4 & KOR & 3.3 & POL & 3.7 & USA & 4.1 \\
\hline 7 & NLD & 3.4 & NLD & 3.4 & HUN & 3.5 & ITA & 2.6 & ITA & 2.7 & JPN & 3.0 & JPN & 3.2 & ESP & 3.6 & NLD & 3.5 \\
\hline 8 & HUN & 2.9 & AUT & 2.6 & CZE & 3.2 & AUT & 2.3 & FRA & 2.4 & CZE & 2.6 & SVK & 2.6 & JPN & 3.0 & BEL & 3.3 \\
\hline 9 & CZE & 2.8 & CZE & 2.5 & KOR & 3.1 & FRA & 2.1 & AUT & 2.4 & ITA & 2.5 & USA & 2.5 & AUT & 2.7 & JPN & 3.3 \\
\hline 10 & USA & 2.7 & HUN & 2.5 & JPN & 2.9 & GBR & 2.1 & GBR & 2.2 & NLD & 2.4 & AUT & 2.4 & SVK & 2.5 & GBR & 3.1 \\
\hline 11 & AUT & 2.5 & POL & 2.2 & SVK & 2.7 & SVK & 2.0 & CZE & 1.7 & USA & 2.1 & GBR & 2.4 & CHN & 2.0 & TUR & 3.1 \\
\hline 12 & GBR & 2.3 & GBR & 2.2 & GBR & 2.4 & POL & 2.0 & THA & 1.7 & AUT & 2.0 & TUR & 2.4 & HUN & 2.0 & NOR & 2.8 \\
\hline 13 & POL & 2.1 & USA & 2.1 & AUT & 2.4 & CZE & 2.0 & MYS & 1.6 & GBR & 1.9 & POL & 2.3 & TUR & 1.6 & SVK & 2.6 \\
\hline 14 & SVK & 2.0 & SVK & 1.5 & ESP & 2.1 & USA & 1.5 & HKG & 1.5 & ROM & 1.6 & BEL & 2.1 & GBR & 1.6 & AUT & 2.0 \\
\hline 15 & ESP & 1.7 & ESP & 1.5 & POL & 2.0 & MYS & 1.5 & POL & 1.4 & FRA & 1.5 & NLD & 2.1 & BEL & 0.9 & HUN & 1.9 \\
\hline 16 & TUR & 1.1 & THA & 1.0 & SGP & 1.6 & HKG & 1.5 & USA & 1.3 & HKG & 1.5 & HUN & 2.0 & USA & 0.8 & LBR & 1.9 \\
\hline 17 & BEL & 1.1 & MYS & 1.0 & BEL & 1.5 & THA & 1.5 & ROM & 1.2 & MYS & 1.4 & NOR & 1.4 & SWE & 0.8 & SWE & 1.7 \\
\hline 18 & SGP & 1.1 & TUR & 0.9 & TUR & 1.5 & ROM & 1.4 & SVK & 1.2 & FIN & 1.4 & CHN & 1.3 & ROM & 0.7 & SGP & 1.5 \\
\hline 19 & THA & 1.1 & ROM & 0.9 & THA & 1.2 & SWE & 0.8 & SGP & 0.8 & IND & 1.1 & SWE & 1.3 & PRT & 0.6 & BHS & 1.4 \\
\hline 20 & MYS & 1.0 & BEL & 0.9 & SWE & 1.2 & ESP & 0.8 & SWE & 0.8 & UKR & 1.1 & LBR & 1.0 & NLD & 0.6 & CAN & 1.3 \\
\hline
\end{tabular}

(Note) see Table A2 for the name of country.

(Source) authors' calculation, using data available from UN comtrade. 
Figure A1. The number of exported product - country pairs

\section{(a) All $\mathrm{m}$}

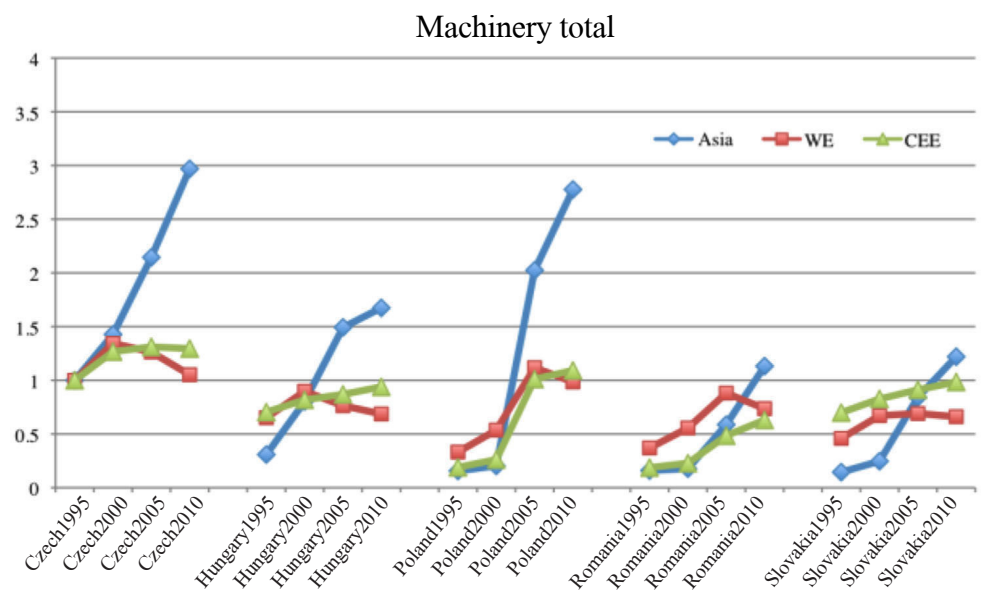

Machinery parts and components

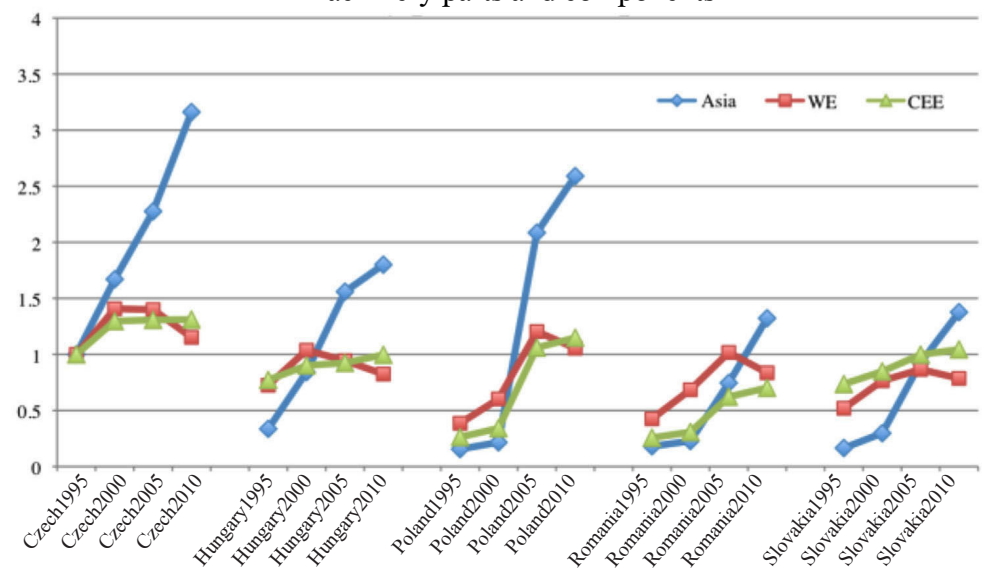

Machinery final products

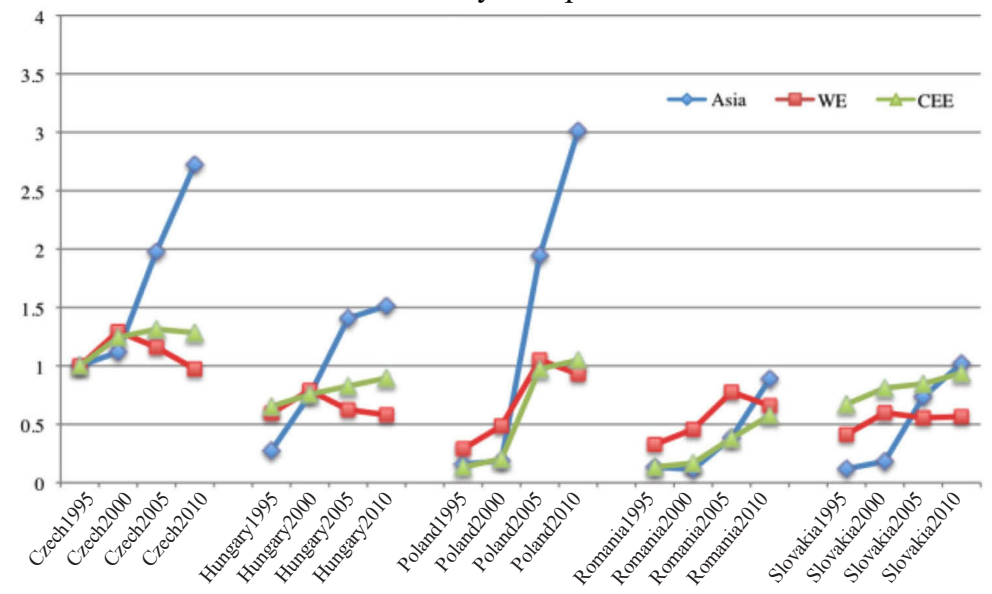




\section{(b) Electric machinery sector}

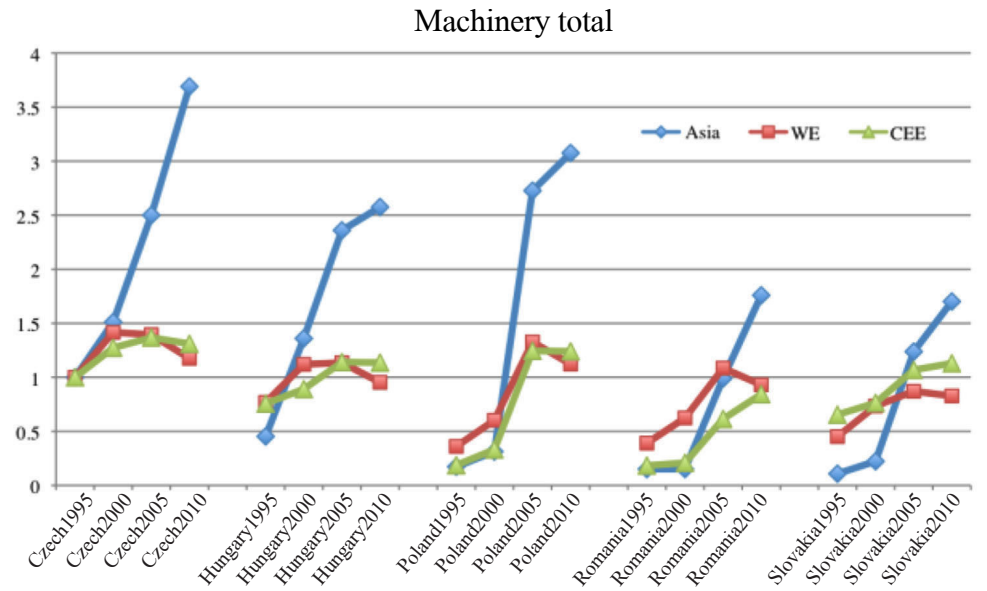

Machinery parts and components

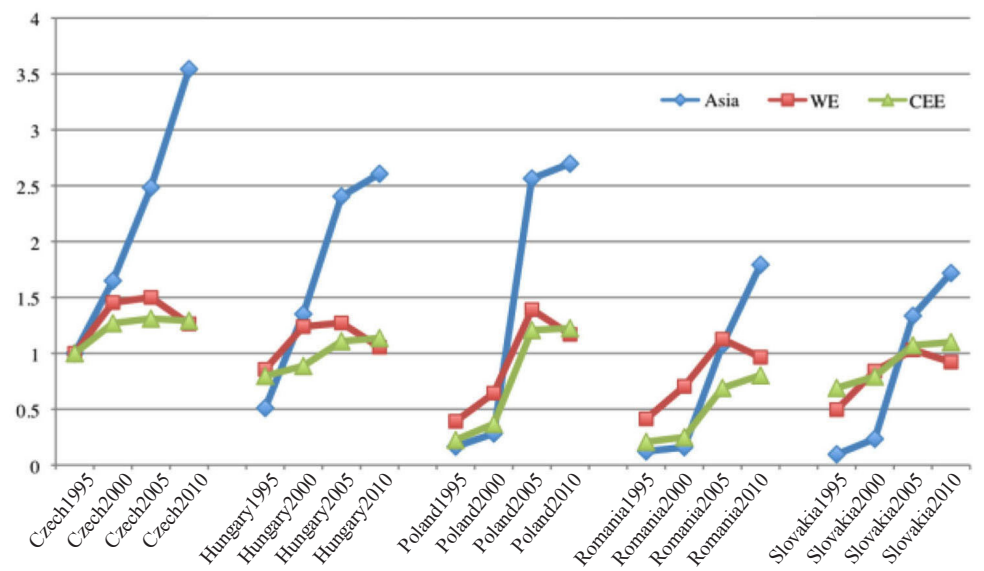

Machinery final products

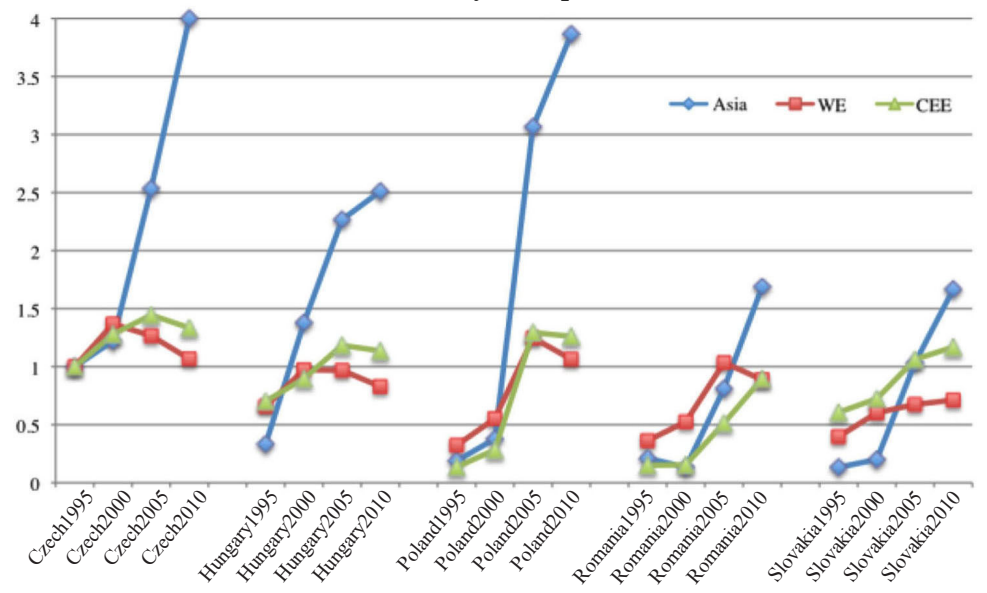




\section{(c) Transport equipment sector}

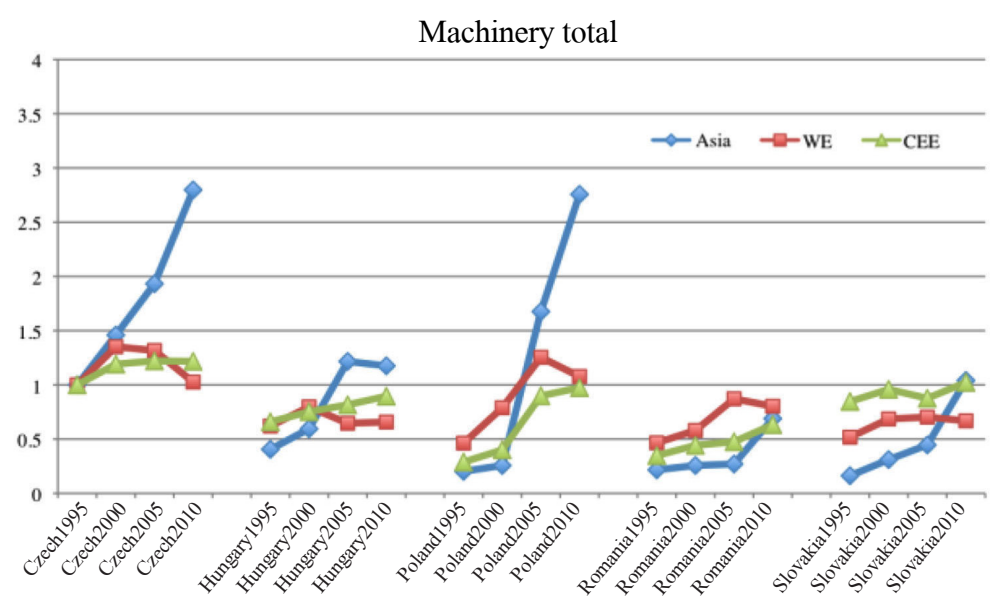

Machinery parts and components

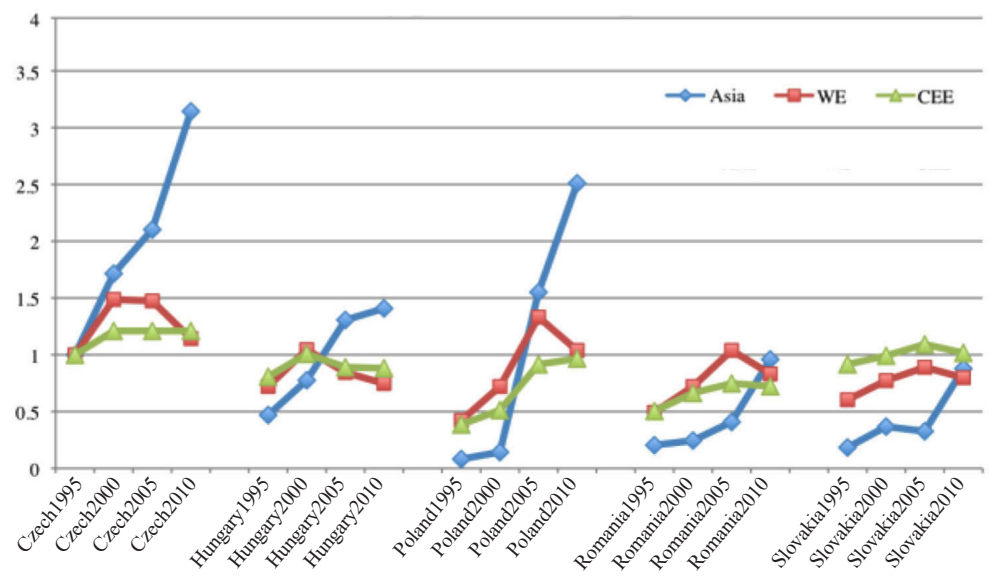

Machinery final products

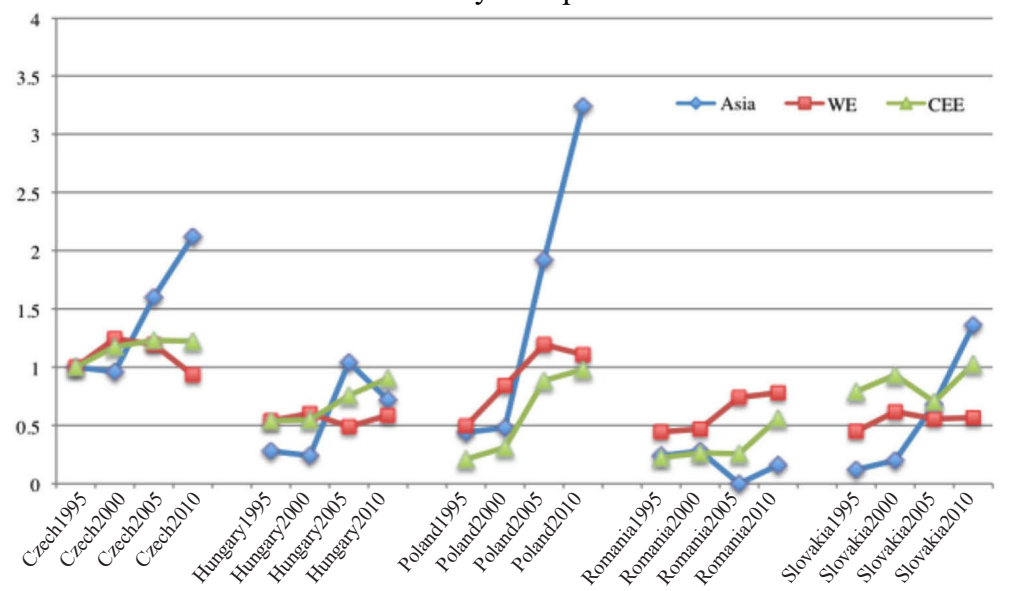

(Note) The number of exported product-country pairs for Czech is as follows: 920(total), 515(parts), and 
405(final products) in all machinery sectors, 277, 188, and 90 in the electric machinery sector, and 74, 49, and 25 in the transport equipment sector for Asia, 3,330, 1,447, and 1,883 in all machinery sectors, 835, 460, and 375 in the electric machinery sector, and 350, 154, and 196 in the transport equipment sector for WE, and 2,422, 1,043, and 1,379 in all machinery sectors, 633, 361, and 272 in the electric machinery sector, and 267, 119, 148 in the transport equipment sector for CEE.

(Source) authors' calculation, using data available from UN comtrade.

Table A2. List of countries

\begin{tabular}{|c|c|c|c|c|c|}
\hline ID & Name & ID & Name & ID & Name \\
\hline ALB & Albania & HKG & "Hong Kong & PAN & Panama \\
\hline DZA & Algeria & HUN & Hungary & PER & Peru \\
\hline ATG & Antigua and Barbuda & ISL & Iceland & PHL & Philippines \\
\hline AZE & Azerbaijan & IDN & Indonesia & POL & Poland \\
\hline ARG & Argentina & ISR & Israel & PRT & Portugal \\
\hline AUS & Australia & ITA & Italy & ROM & Romania \\
\hline AUT & Austria & JPN & Japan & RUS & Russia \\
\hline BHS & Bahamas & KAZ & Kazakhstan & RWA & Rwanda \\
\hline BHR & Bahrain & JOR & Jordan & SAU & Saudi Arabia \\
\hline BGD & Bangladesh & KEN & Kenya & IND & India \\
\hline ARM & Armenia & KOR & Korea & SGP & Singapore \\
\hline BEL & Belgium & KWT & Kuwait & SVK & Slovakia \\
\hline $\mathrm{BIH}$ & Bosnia Herzegovina & LBN & Lebanon & VNM & Viet Nam \\
\hline BRA & Brazil & LVA & Latvia & SVN & Slovenia \\
\hline BGR & Bulgaria & LBR & Liberia & $\mathrm{ZAF}$ & South Africa \\
\hline BLR & Belarus & LTU & Lithuania & ESP & Spain \\
\hline CAN & Canada & LUX & Luxembourg & SDN & Sudan \\
\hline $\mathrm{CHL}$ & Chile & MYS & Malaysia & SWE & Sweden \\
\hline $\mathrm{CHN}$ & China & MLT & Malta & $\mathrm{CHE}$ & Switzerland \\
\hline COL & Colombia & MUS & Mauritius & SYR & Syria \\
\hline CRI & Costa Rica & MEX & Mexico & THA & Thailand \\
\hline HRV & Croatia & MDA & Moldova & ARE & UAE \\
\hline CUB & Cuba & MAR & Morocco & TUN & Tunisia \\
\hline CYP & Cyprus & OMN & Oman & TUR & Turkey \\
\hline DNK & Denmark & NLD & Netherland & UKR & Ukraine \\
\hline EST & Estonia & VUT & Vanuatu & EGY & Egypt \\
\hline FIN & Finland & NZL & New Zealand & GBR & UK \\
\hline FRA & France & NGA & Nigeria & USA & USA \\
\hline GEO & Georgia & NOR & Norway & UZB & Uzbekistan \\
\hline DEU & Germany & MHL & Marshall & VEN & Venezuela \\
\hline GRC & Greece & PAK & Pakistan & YEM & Yemen \\
\hline
\end{tabular}

